\title{
11
}

\section{Jets and Gamma-Ray Burst Unification Schemes}

\author{
Jonathan Granot ${ }^{1}$ and Enrico Ramirez-Ruiz ${ }^{2}$ \\ (1) Centre for Astrophysics Research, University of Hertfordshire, College Lane, \\ Hatfield, Herts, AL10 9AB, UK \\ (2) Astronomy and Astrophysics Department, University of California, \\ Santa Cruz, CA 95064, USA
}

\begin{abstract}
There are several lines of evidence indicating that the ultra-relativistic outflows powering gamma-ray bursts (GRBs) are collimated into narrow jets. However, these are indirect, and the jet structure is rather poorly constrained. What is more, the jet dynamics have still not been investigated in detail. It has been suggested that the observed variety between different long duration events, ranging from bright spectrally hard GRBs, to dimmer and spectrally softer X-ray flashes (XRFs) may be largely due to different viewing angles (or lines of sight) relative to rather similar relativistic jets. Here we describe the current state of knowledge on these topics, explain some of the most relevant physics behind some of the basic principles, and discuss prospects for the future.
\end{abstract}

\subsection{Evidence for bulk relativistic motion in gamma-ray bursts}

The first line of evidence for ultra-relativistic bulk motion of the outflows that produce GRBs arises from the compactness argument. It relies on the observed short and intense pulses of gamma rays and their non-thermal energy spectrum that often extends up to high photon energies. Together, these facts imply that the emitting region must be moving relativistically. In order to understand this better, let us first consider a source that is either at rest or moves at a Newtonian velocity, $\beta \equiv v / c \ll 1$, corresponding to a bulk Lorentz factor $\Gamma \equiv\left(1-\beta^{2}\right)^{-1 / 2} \approx 1$. For such a source the observed variability timescale (e.g., the width of the observed pulses) $\Delta t$, implies a typical source size or radius $R<c \Delta t$, due to light time travel effects (for simplicity we ignore here cosmological effects, such as redshift or time dilation). GRBs often show significant variability down to millisecond timescales, implying $R<3 \times 10^{7}(\Delta t / 1 \mathrm{~ms}) \mathrm{cm}$. At cosmological distances their isotropic 
equivalent luminosity, $L$, is typically in the range of $10^{50}-10^{53} \mathrm{erg} \mathrm{s}^{-1}$. In addition, the (observed part of the) $\varepsilon F_{\varepsilon}$ GRB spectrum typically peaks around a dimensionless photon energy of $\varepsilon \equiv E_{\mathrm{ph}} / m_{e} c^{2} \sim 1$, so that (for a Newtonian source) a good fraction of the total radiated energy is carried by photons that can pair produce with other photons of similar energy. ( $F$ is the radiative flux and $F_{\varepsilon} \equiv \mathrm{d} F / \mathrm{d} \varepsilon$ ). A simple estimate of the opacity to pair production $\left(\gamma \gamma \rightarrow e^{+} e^{-}\right)$usually results in a huge optical depth for this process, $\tau_{\gamma \gamma}(\varepsilon) \sim \sigma_{\mathrm{T}} n_{\gamma}(1 / \varepsilon) R \sim \sigma_{\mathrm{T}} L_{1 / \varepsilon} / 4 \pi m_{e} c^{3} R \gtrsim \sigma_{\mathrm{T}} L_{1 / \varepsilon} / 4 \pi m_{e} c^{4} \Delta t \sim$ $10^{14}\left(L_{1 / \varepsilon} / 10^{51} \mathrm{erg} \mathrm{s}^{-1}\right)(1 \mathrm{~ms} / \Delta t)$, where $L_{\varepsilon} \equiv d L / d \varepsilon$ and $\sigma_{\mathrm{T}}$ is the Thomson cross section (Granot et al. 2008). Such huge optical depths are clearly inconsistent with the non-thermal GRB spectrum, which has a significant power-law high-energy tail. This is known as the compactness problem (Ruderman 1975).

If the source is moving relativistically toward us with a bulk Lorentz factor $\Gamma \gg 1$, then in its own rest frame (where quantities measured in that rest frame are denoted by a prime) the photons have much smaller energies, $\varepsilon^{\prime} \sim$ $\varepsilon / \Gamma$, while in the lab frame (i.e., the rest frame of the central source) most of the photons propagate at angles $\lesssim 1 / \Gamma$ relative to its direction of motion (see Sect. 11.2 on aberration of light). The latter implies that in the lab frame the typical angle between the directions of the interacting photons is $\theta_{12} \sim 1 / \Gamma$, which has the following consequences. First, it increases the threshold for pair production, $\varepsilon_{1} \varepsilon_{2}>2 /\left(1-\cos \theta_{12}\right)$, to $\varepsilon_{1} \varepsilon_{2} \gtrsim \Gamma^{2}$ (compared to $\varepsilon_{1}^{\prime} \varepsilon_{2}^{\prime} \gtrsim 1$ for the roughly isotropic distribution of angles between the directions of the interacting photons in the rest frame of the source, where $\theta_{12}^{\prime} \sim 1$ ). Thus, $L_{1 / \varepsilon}$ needs to be replaced by $L_{\Gamma^{2} / \varepsilon}=\Gamma^{2(1-\alpha)} L_{1 / \varepsilon}$, where $L_{\varepsilon} \approx L_{0} \varepsilon^{1-\alpha}$ at high photon energies (corresponding to $d N_{\mathrm{ph}} / d \varepsilon \propto \varepsilon^{-\alpha}$, i.e., $\alpha$ is the highenergy photon index). This results in an additional factor of $\Gamma^{2(1-\alpha)}$ in the expression for $\tau_{\gamma \gamma}(\varepsilon)$. Second, the expression for the optical depth includes a factor of $1-\cos \theta_{12}$ (that represents the rate at which photons pass each other and have an opportunity to interact) which for a stationary source is $\sim 1$, but for a relativistic source moving toward us is $\sim \Gamma^{-2}$. Finally, the emission radius can be as large as $R \lesssim \Gamma^{2} c \Delta t$, which introduces an additional factor of $\sim \Gamma^{-2}$ in the expression for $\tau_{\gamma \gamma}$. Altogether, $\tau_{\gamma \gamma}(\varepsilon)$ is reduced by a factor of $\sim \Gamma^{2(\alpha+1)}$ (i.e., $\left.\tau_{\gamma \gamma} \propto \Gamma^{-2(\alpha+1)}\right)$. Since typically $\alpha \sim 2-3$, this usually implies $\Gamma \gtrsim 10^{2}$ in order to have $\tau_{\gamma \gamma}<1$ and overcome the compactness problem. Using similar arguments, the lack of a high-energy cutoff due to pair production in the observed spectrum of the prompt $\gamma$-ray emission in GRBs has been used to place lower limits on the Lorentz factor of the outflow (Krolik \& Pier 1991, Fenimore et al. 1993, Woods \& Loeb 1995, Baring \& Harding 1997, Lithwick \& Sari 2001). Recently, high-energy observations 

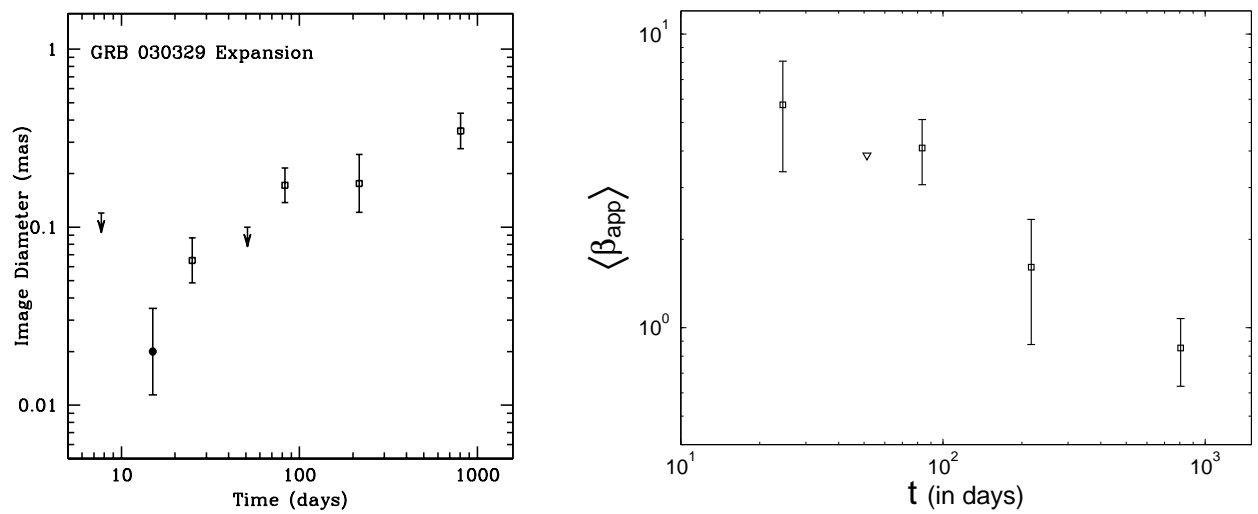

Fig. 11.1. The temporal evolution of the radio afterglow image size of GRB 030329 (left panel) and the implied average apparent expansion velocity in units of $c$ (right panel). (Taken from Pihlström et al. 2007).

by the Fermi Gamma-Ray Space Telescope have enabled particularly strict $\left(\Gamma \gtrsim 10^{3}\right)$ limits to be placed for both long (Abdo et al. 2009a,b) and short (Ackermann et al. 2010) GRBs.

A different, and somewhat complementary, line of evidence for relativistic bulk motion in GRBs comes from estimates of the afterglow image size at relatively late times (weeks to years) in the radio. The afterglow image size increases with time, and therefore it can be (marginally) angularly resolved only at late times, and only for relatively nearby and radio bright afterglows. At such late times the flow is much less relativistic, with a much more modest Lorentz factor. The size of the afterglow image at a single epoch can be estimated from the quenching of diffractive scintillation in the radio afterglow (Goodman 1997), as the angular size of the source becomes larger than that of the relevant density fluctuations in the interstellar medium of our Galaxy, which is estimated to be roughly $\theta_{d} \sim 3 \mu$ as. When the source angular size $\theta_{\mathrm{s}}$ is smaller than $\theta_{d}$ then $\sim\left(\theta_{d} / \theta_{\mathrm{s}}\right)^{2} \gg 1$, and different subimages are formed, which produce a random diffraction pattern resulting in frequency dependent brightness fluctuations of order unity $(\Delta F / F \sim 1)$. For GRB 970508 this implied an apparent source size of $\sim 10^{17} \mathrm{~cm}$ at $t \sim 30$ days, or an average apparent expansion velocity close to the speed of light, $c$, during the first month (Frail et al. 1997, Taylor et al. 1997, Waxman et al. 1998). The flux below the synchrotron self-absorption frequency can also be used to constrain the size of the emitting region (e.g., Katz \& Piran 1997, Granot et al. 2005).

A more direct measurement of the image size, as well as its temporal evolu- 
tion, can be obtained through very long base-line interferometric techniques at radio wavelengths using, e.g., the Very Long Baseline Array (VLBA). This has been possible for only one radio afterglow so far - GRB 030329 at a redshift of $z=0.1685$ (Taylor et al. 2004, 2005, Pihlström et al. 2007), since it requires a bright, relatively nearby event $(z \lesssim 0.2)$. Nevertheless, the measured source size and its temporal evolution imply a relativistic and decelerating apparent expansion velocity (Fig. 11.1), with an average value of $\sim 6 c$ over the first 25 days, and transition to a sub-relativistic expansion after about one year.

\subsection{Aberration of light and the Doppler effect}

In relativistic sources such as GRBs, aberration of light (also known as relativistic beaming) and the Doppler effect play an important role. These two effects refer to the change in direction and frequency, respectively, of electromagnetic waves (or particles - photons) between different frames of reference - in our case between the rest frame of the emitting fluid (that is referred to as the comoving frame), and the lab frame (the rest frame of the central source in which the external medium is at rest and the emitting jet material is moving relativistically). These effects can be easily derived from the Lorentz transformation of the 4-vectors of an electromagnetic wave $k^{\mu}=(\omega / c, \vec{k})$ or from the photon energy-momentum $P^{\mu}=\hbar k^{\mu}=E(1 / c, \hat{k})$. Some more intuition may be gained by the following derivations using the Lorentz transformation: $t=\Gamma\left(t^{\prime}+\beta x^{\prime} / c\right), x=\Gamma\left(x^{\prime}+\beta c t^{\prime}\right), y=y^{\prime}, z=z^{\prime}$. This implies

$$
v_{x} \equiv \frac{d x}{d t}=\frac{d x^{\prime}+\beta c d t^{\prime}}{d t^{\prime}+\beta d x^{\prime} / c}=\frac{v_{x}^{\prime}+\beta c}{1+\beta v_{x}^{\prime} / c} .
$$

For a photon, $v_{x} / c=\cos \theta \equiv \mu$ and $v_{x}^{\prime} / c=\cos \theta^{\prime} \equiv \mu^{\prime}$ are the cosines of the angle between its direction of motion and the $x$-direction (defined as the direction of motion of the primed frame relative to the un-primed frame) in the two rest frames, respectively. Therefore,

$$
\mu=\frac{\mu^{\prime}+\beta}{1+\beta \mu^{\prime}}, \quad \mu^{\prime}=\frac{\mu-\beta}{1-\beta \mu},
$$

where the second equality is derived from symmetry (interchanging primed and un-primed quantities and replacing $\beta$ by $-\beta$ ). This is the formula for aberration of light (or relativistic beaming). It is demonstrated in Fig. 11.2 for a point source emitting isotropically in its own rest frame. For a relativistic source moving with a Lorentz factor $\Gamma=\left(1-\beta^{2}\right)^{-1 / 2} \gg 1$ (in the 

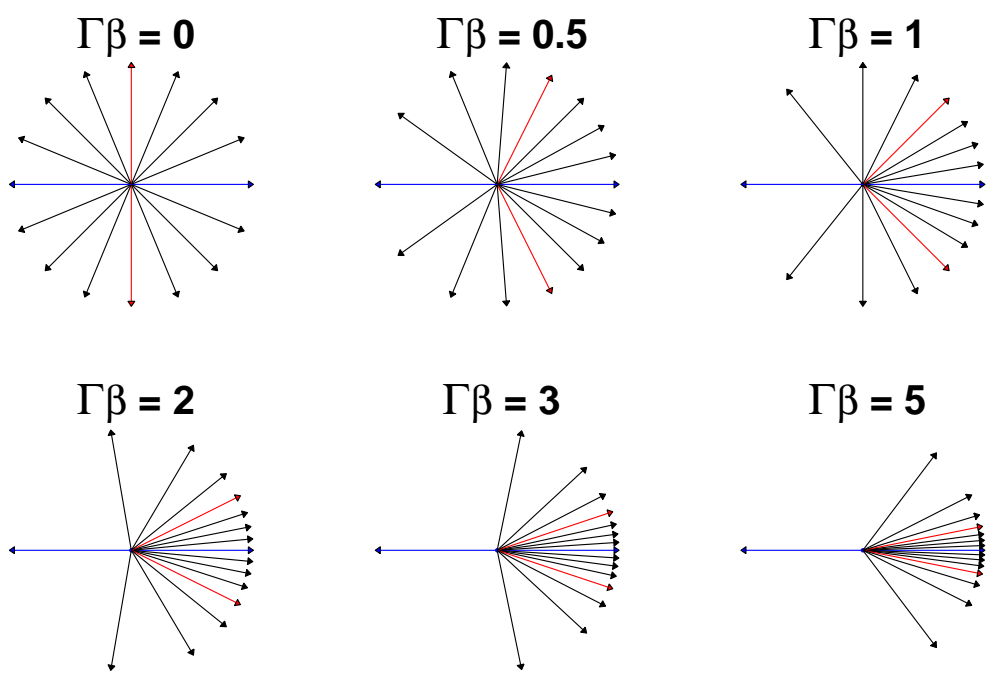

Fig. 11.2. Aberration of light: the arrows show the directions of photons in the lab frame for a point source that emits isotropically in its own rest frame and moves to the right at different values of the four-velocity $\Gamma \beta$. For $\Gamma \gg 1$, half of the photons (and $3 / 4$ of the radiated energy) are within an angle of $1 / \Gamma$ around the source's direction of motion (between the grey arrows, which correspond to $\theta^{\prime}=90^{\circ}$ ).

lab frame), half of the photons and most (3/4) of the emitted energy are within an angle of $1 / \Gamma$ around its direction of motion.

The formula for the Doppler factor, $\mathcal{D} \equiv \nu / \nu^{\prime}$, can be derived noticing that the phase of an electromagnetic wave must be Lorentz invariant, since if the electric and magnetic fields vanish in one frame then they must vanish in all frames. In particular, over one period of oscillation of the electromagnetic field, $P=1 / \nu=\lambda / c$, a photon travels a distance equal to its wavelength $\lambda$, and similarly $P^{\prime}=1 / \nu^{\prime}=\lambda^{\prime} / c$, so that $\mathcal{D}=\lambda^{\prime} / \lambda$. Note that because of this simple consideration $\lambda$ must be equal to the difference in the path length to the observer (measured simultaneously in the lab frame) between two hypothetical photons emitted with a single period time difference (i.e., $c$ times the difference in the arrival time to the observer of such hypothetical photons). Over a single period, a time $P^{\prime}=\lambda^{\prime} / c$ elapses in the comoving frame, which due to time dilation corresponds to a time $\Delta t_{\text {lab }}=\Gamma \lambda^{\prime} / c$ in the lab frame over which the source travels a distance of $l=\beta c \Delta t_{\text {lab }}=\Gamma \beta \lambda^{\prime}$. During the same time the first hypothetical photon has traveled a distance of $c \Delta t_{\text {lab }}=\Gamma \lambda^{\prime}$, which implies, from simple geometrical arguments, that $\lambda=\Gamma \lambda^{\prime}-l \mu=\lambda^{\prime} \Gamma(1-\beta \mu)$ and therefore $\mathcal{D}=\lambda^{\prime} / \lambda=1 / \Gamma(1-\beta \mu)$.

Due to symmetry considerations similar to those mentioned above, $\mathcal{D}^{\prime} \equiv$ 
$\nu^{\prime} / \nu=1 / \Gamma\left(1+\beta \mu^{\prime}\right)$ and therefore $\mathcal{D}=1 / \mathcal{D}^{\prime}=\Gamma\left(1+\beta \mu^{\prime}\right)$. In particular, $1 / \Gamma(1-\beta \mu)=\Gamma\left(1+\beta \mu^{\prime}\right)$, which is equivalent to eq. 11.2. Expressing $\mathcal{D}$ in the comoving frame makes it easier to calculate various quantities for an isotropic emitter in this frame, which is broadly expected in most cases of astrophysical interest. For example, it is easy to see that the average Doppler factor for such an isotropic emitter is simply $\Gamma$, while a fraction $(2+\beta) / 4$ of the energy in the lab frame is carried by photons with $\theta^{\prime}<90^{\circ}$ that correspond to $\cos \theta>\beta$ (or $\theta<1 / \Gamma$ for $\Gamma \gg 1$ ).

Finally, we note that due to symmetry (no preferred direction in the plane normal to $\vec{\beta}=\vec{v} / c$ ), the azimuthal angle of the photon direction of motion is equal in the two frames, $\phi=\phi^{\prime}$, and thus the differential solid angles $d \Omega=d \phi d \mu$ and $d \Omega^{\prime}=d \phi^{\prime} d \mu^{\prime}$ are related by $d \Omega^{\prime} / d \Omega=d \mu^{\prime} / d \mu=\mathcal{D}^{2}$, using eq. 11.2 .

\subsection{Evidence for jets in gamma-ray bursts}

In contrast to Active Galactic Nuclei (AGN) and Galactic micro-quasars, in many of which the relativistic jets are well resolved and their structure can be studied directly, the images of GRBs are usually unresolved, or at best the image of their late time radio afterglow is only marginally resolved (i.e., the image size is still somewhat smaller than the instrumental beam size, and its size and shape can be only rather crudely estimated). Therefore, the evidence for jets (i.e., highly collimated outflows) in GRBs is mainly indirect. The main arguments, or lines of evidence, in favor of jets in GRBs are as follows.

First, other known sources of relativistic outflows (such as micro-quasars and AGN) are collimated into narrow jets, so it is natural to expect a similar behavior for the relativistic outflow in GRBs, if indeed the underlying process for launching jets are similar (e.g., accretion onto a black hole).

Second, the very high values deduced for the energy output in gamma rays assuming isotropic emission, $E_{\gamma, \text { iso }}$, that are inferred for GRBs with known redshifts, approach and in some cases even exceed $M_{\odot} c^{2}$ (for GRB 080916C $E_{\gamma, \text { iso }} \approx 4.9 M_{\odot} c^{2}$; Abdo et al. 2009a). Such extreme energies in an ultrarelativistic outflow are hard to envision in models involving stellar mass progenitors. If the outflow is collimated into a narrow jet (or bipolar jets) that occupies a small fraction $f_{b} \ll 1$ of the total solid angle, then the strong relativistic beaming due to the very high initial Lorentz factor $\left(\Gamma_{0} \gtrsim 100\right)$ will cause the emitted gamma rays to be similarly collimated. This reduces the true energy output in gamma rays by a factor of $f_{b}^{-1}$ to $E_{\gamma}=f_{b} E_{\gamma \text {,iso }}$, thus significantly reducing the energy requirements (Rhoads 1997, Halpern 
et al. 1999, Sari et al. 1999). In addition, there is good (spectroscopic) evidence that at least some GRBs of the long-soft class (Kouveliotou et al. 1993) are coincident (to within a few days) with a core collapse supernova belonging to the Type Ic category (Stanek et al. 2003, Hjorth et al. 2003). In such cases the average Lorentz factor must be $\langle\Gamma\rangle \lesssim 2$ for a spherical explosion, since the accreted mass is not expected to significantly exceed the ejected mass, and only a fraction of the rest energy of the former can provide the kinetic energy for the latter. Therefore, only a minute fraction of the ejected mass can reach $\Gamma \gtrsim 100$ that is required in order to power the GRB. Hydrodynamic analysis of spherical blastwaves (Tan et al. 2001, Perna \& Vietri 2002) shows that material with $\Gamma \gtrsim 100$ would carry only a small fraction of the total energy, insufficient to account for the high observed values of $E_{\gamma \text {,iso }}$. This is because the energy in the explosion is deposited into the bulk of the ejecta, and only a decreasing fraction of the total energy is transfered to a smaller fraction of the ejected mass that is accelerated to subsequently higher velocities as the supernova shock propagates into the steep density gradient at the outer edge of the progenitor star. For a jet the ejected mass can be much smaller than the accreted mass so that $\langle\Gamma\rangle \gg 1$ is possible, and a good fraction of the total energy in the outflow may be deposited in material with $\Gamma \gtrsim 100$ that can power the GRB. Moreover, the energy requirements for a jet are much less severe, since the same observed value of $E_{\gamma, \text { iso }}$ implies a much smaller beaming corrected radiated energy $E_{\gamma}$, so that typically only a small fraction $\left(\lesssim 10^{-3}-10^{-2}\right)$ of the total available energy is required to power the jet.

Finally, a somewhat more direct line of evidence in favor of a narrowly collimated outflow comes from achromatic breaks seen in the afterglow light curves of many GRBs (Fruchter et al. 1999, Kulkarni et al. 1999, Harrison et al. 1999, Halpern et al. 2000, Price et al. 2001, Sagar et al. 2001) $\oplus$ In fact, such a "jet break" in the afterglow light curve was predicted before it was observed (Rhoads 1997, 1999, Sari et al. 1999). The exact cause of the jet break is discussed in detail in Sect. 11.8. A brief explanation follows. Due to relativistic beaming (and quasi-radial velocities of the emitting material) most of the observed emission comes from a visible region of angle $\sim 1 / \Gamma$ around our line of sight. As the jet decelerates by sweeping-up the external medium, the angular size of the visible region increases, until the edge of the jet becomes visible when $\Gamma$ drops below the inverse of its half-opening angle, and from this point onward the observer "misses" the flux from outside the

$\dagger$ Somewhat earlier, GRB 980519 showed a steep afterglow flux decay rate $\left(\sim t^{-2}\right)$, which was interpreted as being due an earlier (unseen) jet break (Halpern et al. 1999) 
edge of the jet, which would have been present if the flow were spherical, resulting in a faster flux decay (i.e., a jet break).

For a bipolar jet that is uniform within its initial half-opening angle, $\theta_{0}$, the collimation or beaming factor is given by $f_{b}=1-\cos \theta_{0} \approx \theta_{0}^{2} / 2$, and values of $f_{b} \sim 10^{-3}-10^{-1}$ (corresponding to $\theta_{0}$ values ranging between a few degrees and a few tens of degrees) have been derived in the pre-Swift era from such jet breaks (e.g., Frail et al. 2001). However, over the first years of Swift operations, only a handful of convincing jet-break candidates were identified (Blustin et al. 2006, Stanek et al. 2007, Willingale et al. 2007, Kocevski \& Butler 2008), leading to concerns about the viability of this picture. Since then, however, deep optical and X-ray observations have revealed evidence for jet breaks in several additional Swift afterglows (Dai et al. 2008; Liang et al. 2008; Racusin et al. 2009; Burrows et al. 2009; Tanvir et al. 2010). A significant fraction of these aftreglows were monitored to late times, suggesting that jet breaks for typical Swift GRBs may be occurring at late times and faint flux levels that are beyond the limit of the standard ground- and space-based campaigns. This, in turn, might be attributed to Swift's higher sensitivity compared to previous instruments, which results in the detection of fainter GRBs that (on average) correspond to wider jets.

\subsection{The jet structure}

The lack of well resolved GRB images makes it very difficult for the jet structure to be deduced. The initial Lorentz factor during the prompt gamma-ray emission is very high, $\Gamma_{0} \gtrsim 100$, and therefore we observe emission mainly from very small angles, $\theta \lesssim \Gamma_{0}^{-1} \lesssim 10^{-2} \mathrm{rad}$, relative to our line of sight. This is a result of relativistic beaming (i.e., aberration of light; see Sect. 11.2). For this reason, the prompt gamma-ray emission probes only a very small region of solid angle $\sim \pi \Gamma_{0}^{-2}$, or a fraction $\sim \Gamma_{0}^{-2} / 4 \sim 10^{-7}-10^{-4.5}$ of the total solid angle. Thus, the prompt emission provides no information about the ejecta that are moving in other directions (i.e., at angles $\gg \Gamma_{0}^{-1}$ from our direction): the outflow could be spherical, or concentrated in a conical jet of half-opening angle $\theta_{0}>\Gamma_{0}^{-1}$ (provided that our line of sight is inside the cone, at an angle of $\gtrsim \Gamma_{0}^{-1}$ from its edge).

During the afterglow, however, the Lorentz factor $\Gamma$ of the emitting material decreases with time, since the afterglow shock decelerates as it sweeps up the external medium. This causes the beaming (or light aberration) effects to gradually become less severe, as $\Gamma$ becomes more moderate, so that we observe afterglow emission from a wider range of angles (of $\lesssim \Gamma^{-1}$ from our line of sight, and not just from material moving almost directly towards 
us, at angles $\lesssim \Gamma_{0}^{-1}$, as in the prompt emission). This enables us to probe the jet structure over increasingly larger angular scales.

For a jet with axial symmetry, its structure $\dagger$ can be described by the distribution of its total energy (excluding rest energy) content per solid angle, $\mathcal{E}$, and initial Lorentz factor $\Gamma_{0}$, as a function of the jet's polar angle $\theta$. We note that $\Gamma_{0}(\theta)$ affects mainly the prompt gamma-ray emission and early afterglow, since it is largely "forgotten" after the local deceleration time $t_{\mathrm{dec}}(\theta) \sim R_{\mathrm{dec}}(\theta) / 2 c \Gamma_{0}^{2}(\theta)$, while $\mathcal{E}(\theta)$ affects also the late time afterglow emission. Here $R_{\mathrm{dec}}(\theta)$ is the local deceleration radius where most of the energy in the outflow at an angle $\theta$ is transferred to the shocked external medium; for a power-law external density, $\rho_{\text {ext }}(r)=A r^{-k}$, it is given by $⿴$ $R_{\mathrm{dec}}(\theta) \approx\left[(3-k) \mathcal{E}(\theta) / A c^{2} \Gamma_{0}^{2}(\theta)\right]^{1 /(3-k)}$. The structure of GRB jets is very important for deducing their event rate and total energy, as well as for requirements on the jet production mechanisms.

The most popular model for the structure of GRB jets is the uniform jet (UJ, or "top hat") model (Rhoads 1997, 1999, Panaitescu \& Mészáros 1999, Sari et al. 1999, Kumar \& Panaitescu 2000, Moderski et al. 2000, Granot et al. 2001, 2002, Ramirez-Ruiz \& Madau 2004, Ramirez-Ruiz et al. 2005), where $\mathcal{E}$ and $\Gamma_{0}$ are uniform within some finite half-opening angle, $\theta_{\mathrm{j}}$, and sharply drop outside of $\theta_{\mathrm{j}}$. An alternative jet structure that is also rather popular is the universal structured jet (USJ) model (Mészáros et al. 1998, Lipunov et al. 2001, Rossi et al. 2002, Zhang \& Mészáros 2002), where $\mathcal{E}$ and $\Gamma_{0}$ vary smoothly with $\theta$, as a power law outside of some narrow core angle, typically with equal energy per decade in $\theta, \mathcal{E} \propto \theta^{-2}$. In the UJ model the different values of the jet break time, $t_{\mathrm{j}}$, in the afterglow light curve arise mainly due to different $\theta_{\mathrm{j}}$ (and to a lesser extent due to different ambient densities). In the USJ model, all GRB jets are assumed to be identical, and the different values of $t_{\mathrm{j}}$ arise mainly due to different viewing angles, $\theta_{\mathrm{obs}}$, from the jet axis. In fact, the expression for $t_{\mathrm{j}}$ is similar to that for a uniform jet with $\mathcal{E} \rightarrow \mathcal{E}\left(\theta=\theta_{\text {obs }}\right)$ and $\theta_{\mathrm{j}} \rightarrow \theta_{\text {obs }}$.

An alternative jet structure that has been proposed in the literature is one with a Gaussian angular profile (Zhang \& Mészáros 2002, Kumar \& Granot 2003). It may be thought of as a more realistic version of a uniform jet, where the edges are smooth rather than sharp. A Gaussian jet, $\mathcal{E}(\theta) \propto \exp \left(-\theta^{2} / 2 \theta_{c}^{2}\right)$, is approximately intermediate between the UJ and USJ models. However, it is closer to the UJ model than to the USJ model with $\mathcal{E} \propto \theta^{-2}$ in the sense that the energy in the wings of a Gaussian jet

$\dagger$ We refer here to the angular structure, and ignore the radial structure of the outflow, which becomes unimportant once most of the energy is transfered to the shocked external medium.

$\ddagger$ This expression assumes a "thin" shell, as discussed in Sect. 11.5 
is much smaller than in its core, whereas for a USJ with $\mathcal{E} \propto \theta^{-2}$ wings there is equal energy per decade (in $\theta$ ) in the wings, and therefore the wings contain more energy than the core (by about an order of magnitude).

Another jet structure that is gradually receiving more attention is a twocomponent jet (Pedersen et al. 1998, Frail et al. 2000, Berger et al. 2003, Huang et al. 2004, Peng et al. 2005, Racusin et al. 2008) with a narrow uniform jet of initial Lorentz factor $\Gamma_{0} \gtrsim 100$ surrounded by a wider uniform jet with $\Gamma_{0} \sim 10-30$. Such a jet structure was predicted in the context of the cocoon in the collapsar model (Ramirez-Ruiz et al. 2002) and in the context of a hydromagnetically driven neutron-rich jet (Vlahakis et al. 2003). This model has been invoked in order to account for sharp bumps (i.e., fast rebrightening episodes) in the afterglow light curves of GRB 030329 (Berger et al. 2003) and XRF 030723 (Huang et al. 2004), but detailed calculations show that it cannot produce very sharp features in the light curve (Granot 2005). A different motivation for such jet structure comes about from the energetics of GRBs and X-ray flashes, which could help reduce the high efficiency requirements from the prompt gamma-ray emission (Peng et al. 2005). Later Swift observations (e.g., Nousek et al. 2006) showed that while it can reproduce the early X-ray afterglow light curves, and specifically the shallow decay phase, it does not significantly help reduce the required gamma-ray efficiency (Granot et al. 2006). A different jet structure that has been suggested is that of a jet with a cross section in the shape of a "ring," sometimes referred to as a "hollow cone" (Levinson \& Eichler 1993, 2000, Eichler \& Levinson 2003, 2004, Lazzati \& Begelman 2005), which is uniform within $\theta_{c}<\theta<\theta_{c}+\Delta \theta$ where $\Delta \theta \ll \theta_{c}$.

Finally, it has been argued that there might be random variations on small angular scales in the prompt GRB brightness and in the energy per solid angle in the jet (and therefore also in the corresponding afterglow brightness), around some uniform mean value - the "patchy shell" model (Kumar \& Piran 2000). This model predicts wide variations in $E_{\gamma \text {,iso }}$ between different lines of sight relative to the same GRB jet, as well as fluctuations in the afterglow light curve whose amplitude decreases with time as the visible region (of angle $\lesssim \Gamma^{-1}$ around the line of sight) increases, thus effectively averaging the emission over an increasing number of bright and dim regions. The afterglow flux for different lines of sight approaches the same value at late times, thus implying a relatively narrow distribution of the afterglow (isotropic equivalent) luminosity compared to $E_{\gamma \text {,iso }}$, and no obvious correlation with $E_{\gamma, \text { iso. }}$. While this seemed plausible at the time, later Swift observations showed a clear correlation where GRBs with large $E_{\gamma \text {,iso }}$ tend to have a more luminous afterglow emission (Nousek et al. 2006, Gehrels 
et al. 2008). A more extreme version of the "patchy shell" model is the "mini-jets" model (Yamazaki et al. 2004), where the regions of bright emission and high energy per solid angle are considered as discrete mini-jets, and the regions between them are assumed to have a negligible emission or energy per solid-angle. The main difference is that in this model many lines of sight fall between the mini-jets and the early emission is dominated by the one or more mini-jets closest to the line of sight, whose beaming cone initially does not encompass the line of sight. The original motivation for this model was unifying short GRBs with long GRBs and XRFs, where long GRBs, short GRBs and XRFs correspond to lines of sight with many, one and no mini-jets, respectively. However, the later discovery that the host galaxies of short GRBs are a different population from those of long GRBs (Gehrels et al. 2005, see also Fong et al. 2010) rules out the inclusion of short GRBs in such a unification scheme.

Constraining the jet structure: efforts have been made to constrain the jet structure through statistical studies, linear polarization, afterglow light curves, off-axis viewing and orphan afterglows. Statistical studies have focused on the $\log N-\log S$ distribution where $N$ is the number of GRBs above some threshold peak photon flux $S$ (e.g., Firmani et al. 2004, Guetta et al. 2005), as well as on $d N / d \theta$ (Perna et al. 2003) or $d N / d z d \theta$ (Nakar et al. 2004), where $z$ is the redshift and $\theta$ is $\theta_{\mathrm{j}}$ for the UJ model or $\theta_{\text {obs }}$ for the USJ model, and is determined from the jet break time in the afterglow light curve. These studies were inconclusive, though they showed some preference for the UJ model over the USJ model. The same holds for studies involving the shape of afterglow light curves (Granot \& Kumar 2003), but nevertheless some jets structures could be ruled out using this method (Granot 2005). Studies based on afterglow polarization evolution need to deconvolve the effects of the jet structure from those of the magnetic field configuration in the post-shock emitting region, which make it difficult to draw strong conclusions about the jet structure without making similarly strong assumptions about the structure of the magnetic field itself.

Orphan afterglows are events in which the late time afterglow is detected while the prompt gamma-ray emission is not. Unfortunately, no such events have been clearly observed so far, and the upper limits on the rates of orphan afterglows are still not very constraining for most of the different jet structures. Nevertheless, upper limits on the fraction of $\mathrm{SN} \mathrm{Ib/c} \mathrm{that} \mathrm{show}$ late time radio emission of possible afterglow origin (Soderberg et al. 2006) already argue quite strongly against the presence of energetic, narrow jets (of half-opening angle $\theta_{0} \sim 1 / \Gamma_{0} \sim 10^{-2.5}$ ) which would imply that a large 
fraction $\mathrm{SNe} \mathrm{Ib} / \mathrm{c}$ should harbor GRB jets (most of which point away from us and can be detected only at late times in the radio, when the jets become sub-relativistic). Such extremely narrow jets would also imply a very low true energy, which would be inconsistent with the lower limits on the kinetic energy inferred from late time radio afterglow observations (Berger, Kulkarni \& Frail 2004, Frail et al. 2005). On the other hand, two Type Ic supernovae have been recently observed to harbor relativistic jets (SN 2007gr - Paragi et al. 2010, and SN 2009bb - Soderberg et al. 2010), albeit with an energy either much (SN 2007gr) or slightly (SN 2009bb) lower than is needed to produce a typical bright GRB. These two supernovae amount to about $\sim 1 \%$ of relatively nearby Type Ib/c supernovae, for which a search for such jets has been performed at radio wavelengths.

\subsection{Dynamics of gamma-ray burst outflows}

Here we provide a brief summary of the key aspects for the dynamics of the interaction between the GRB outflow and the surrounding medium, and provide a simple intuitive explanation for some of the most important results. We begin with the relatively simpler case of a spherical outflow, and consider a uniform unmagnetized shell of ejecta of initial width $\Delta_{0}$ and Lorentz factor $\Gamma_{0}$ in the lab frame, propagating into an external density $\rho_{1}(R)$. A forward shock is driven into the ambient medium, while the ejecta are decelerated by a reverse shock. Thus four regions exist: (1) unshocked external medium, (2) shocked external medium, (3) shocked ejecta, and (4) unshocked (freely expanding) ejecta. All the velocities are measured relative to region 1, while the pressure $p$ and rest mass density $\rho$ (or number density $n$ ) are measured in the fluid rest frame. A subscript $i$ between 1 and 4 refers to region $i$, while a subscript $i j$ refers to the relative velocity of regions $i$ and $j$, so that $\Gamma_{i 1}=\Gamma_{1 i}=\Gamma_{i}$. Given $\Gamma_{4}=\Gamma_{0}, \Gamma_{1}=1, \rho_{4}, \rho_{1}$, and assuming the shell of ejecta and external medium are both cold $\left(p_{i} \ll \rho_{i} c^{2}\right.$ for $\left.i=1,4\right)$, there are 8 unknown hydrodynamic quantities $\left(\rho_{2}, p_{2}, \Gamma_{2}, \rho_{3}, p_{3}, \Gamma_{3}\right.$, and the Lorentz factors of the forward and reverse shock fronts) that can be found from the conditions across the contact discontinuity separating regions 2 and 3 $\left(p_{2}=p_{3}\right.$ and $\left.\Gamma_{2}=\Gamma_{3} \equiv \Gamma\right)$ as well as the shock jump conditions (continuity of the energy, momentum, and particle fluxes) across the forward and reverse shocks (between regions $1 \& 2$, and $4 \& 3$, respectively). For simplicity, this is treated in planar symmetry, and the spherical nature of the flow enters only when the evolution of the flow with radius is considered.

An approximate, order of magnitude, estimate of the dynamics at this stage can be obtained by equating the ram pressure of the incoming fluid 
from regions 4 and 1 , as seen from the contact discontinuity (the rest frame of regions 2 and 3): $\rho_{1} u_{21}^{2} \sim \rho_{4} u_{34}^{2}$, where $u=\Gamma \beta$ is the 4-velocity. For $\rho_{4}=\rho_{1}$ we must have $u_{21}=u_{34}$ due to symmetry, and for $\Gamma_{4}=\Gamma_{0} \gg 1$ this implies that both the forward and reverse shocks are relativistic, $u_{21}=$ $u_{43}=\left[\left(\Gamma_{4}-1\right) / 2\right]^{1 / 2} \approx\left(\Gamma_{4} / 2\right)^{1 / 2} \gg 1$. As long as the forward shock is relativistic, $1 \ll \Gamma_{3}=\Gamma_{2} \approx u_{21} \sim u_{34}\left(\rho_{4} / \rho_{1}\right)^{1 / 2}$; when the reverse shock is relativistic then $1 \ll u_{43} \approx \Gamma_{43} \approx \Gamma_{4} / 2 \Gamma_{3}$, so that $\Gamma_{2} \approx\left(\Gamma_{0} / 2\right)^{1 / 2}\left(\rho_{4} / \rho_{1}\right)^{1 / 4}$ and $\Gamma_{34} \approx\left(\Gamma_{0} / 2\right)^{1 / 2}\left(\rho_{1} / \rho_{4}\right)^{1 / 4}$ (Sari \& Piran 1995). Therefore, the condition for the forward shock to be relativistic is $\Gamma_{0}^{2} \gg \rho_{1} / \rho_{4}$, which is typically always satisfied (until very late times when the flow becomes Newtonian), so that $u_{21} \approx \Gamma_{2}$ and $u_{43} \sim \Gamma_{2}\left(\rho_{1} / \rho_{4}\right)^{1 / 2}$. The condition for the reverse shock to be relativistic is $\Gamma_{0}^{2} \gg \rho_{4} / \rho_{1}$. The revere shock is Newtonian in the opposite limit, $\Gamma_{0}^{2} \ll \rho_{4} / \rho_{1}$, and, in this case, $\Gamma_{2} \approx \Gamma_{0}$ so that $u_{43} \sim \Gamma_{0}\left(\rho_{1} / \rho_{4}\right)^{1 / 2} \ll 1$.

Note that $u_{43} \sim \min \left(a^{1 / 4}, a^{1 / 2}\right)$ where $a=\Gamma_{0}^{2} \rho_{1} / \rho_{4} \sim A c^{2} \Gamma_{0}^{4} R^{2-k} \Delta / E \sim$ $\Gamma_{0}^{4} R^{2-k} \Delta / l^{3-k}$ for a power-law external density, $\rho_{1}=A R^{-k}$, where $l \sim$ $\left(E / A c^{2}\right)^{1 /(3-k)}$ is the Sedov length. For a narrow distribution of Lorentz factors $\Delta \approx \Delta_{0}=$ const, and therefore $a \propto R^{2-k}$, i.e., for $k<2$ the reverse shock is initially Newtonian and strengthens with radius. If there is a reasonable spread in the Lorentz factor of the outflow, $\Delta \Gamma_{0} \sim \Gamma_{0}$, then the shell can spread, $\Delta \sim \max \left(\Delta_{0}, R / \Gamma_{0}^{2}\right)$, where $\Delta \propto R$ and therefore $a \propto R^{3-k}$ at $R>R_{\mathrm{s}} \sim \Gamma_{0}^{2} \Delta_{0}$ so that then the reverse shock strengthens for $k<3$.

If the reverse shock is relativistic by the time it finishes crossing the shell, then most of the energy is transfered to the shocked external medium within a single shell crossing. If there is only a very small spread in $\Gamma_{0}$ then the reverse shock can still be Newtonian when it finishes crossing the shell. In this case a large number of Newtonian shocks and rarefaction waves may need to cross the shell before most of the energy is transfered to the shocked external medium (Sari 1997). However, if there is a reasonable spread in the Lorentz factor of the outflow, $\Delta \Gamma_{0} \sim \Gamma_{0}$, then the shell starts to spread before the reverse shock finishes crossing it, in such a way that by the time it crosses the shell the reverse shock already becomes mildly relativistic, so that most of the energy is transfered to the shocked external medium in a single shell crossing time (Sari \& Piran 1995). The dividing line between these two cases corresponds to $1=a\left(R_{\mathrm{S}}\right) \sim \Gamma_{0}^{2(4-k)}\left(\Delta_{0} / l\right)^{3-k}$, where $a\left(R_{\mathrm{S}}\right)>1$ implies a relativistic reverse shock or a "thick" shell and $a\left(R_{\mathrm{S}}\right)<1$ implies a Newtonian reverse shock (without spreading) or a "thin" shell.

Most of the energy is transferred to the shocked external medium at a 
radius $R_{\mathrm{dec}} \sim l \Gamma_{\mathrm{dec}}^{-2 /(3-k)}$ where $\Gamma_{\mathrm{dec}}=\Gamma\left(R_{\mathrm{dec}}\right) \sim \min \left[\Gamma_{0}, \Gamma_{\mathrm{cr}}\right]$ and

$$
\Gamma_{\mathrm{cr}}=\left(\frac{l}{\Delta_{0}}\right)^{(3-k) / 2(4-k)}= \begin{cases}280 \zeta^{3 / 8} E_{53}^{1 / 8} n_{0}^{-1 / 8} T_{50}^{-3 / 8} & (k=0), \\ 70 \zeta^{1 / 4} E_{53}^{1 / 4} A_{*}^{-1 / 4} T_{50}^{-1 / 4} & (k=2),\end{cases}
$$

where $\zeta=(1+z) / 2, T_{\mathrm{GRB}}=(1+z) \Delta_{0} / c=50 T_{50} \mathrm{~s}$ is the duration of the GRB, $E=10^{53} E_{53}$ erg is the (isotropic equivalent) energy of the flow, and $n=n_{0} \mathrm{~cm}^{-3}=A / m_{p}$ for $k=0$, while $A_{*}=A /\left(5 \times 10^{11} \mathrm{gr} \mathrm{cm}^{-1}\right)$ for $k=2$. For $\Gamma_{0}>\Gamma_{\text {cr }}$ we have a thick shell or relativistic reverse shock, and the observed deceleration time is similar to the duration of the GRB, $t_{\mathrm{dec}} \sim$ $R_{\mathrm{dec}} / 2 c \Gamma_{\mathrm{dec}}^{2} \sim(1+z) \Delta_{0} / c \sim T_{\mathrm{GRB}}$. For $\Gamma_{0}<\Gamma_{\mathrm{cr}}$ we have a Newtonian (or at most mildly relativistic) reverse shock or a thin shell, and in this case $t_{\mathrm{dec}} \sim(l / c) \Gamma_{0}^{-2(4-k) /(3-k)}>T_{\mathrm{GRB}}$ (where $l / c \sim t_{\mathrm{NR}}$ is the non-relativistic transition time) and is given by

$$
t_{\mathrm{dec}}=(1+z) \frac{R_{\mathrm{dec}}}{2 c \Gamma_{0}^{2}}=\left\{\begin{array}{cc}
18 \zeta E_{53}^{1 / 3} n_{0}^{-1 / 3}\left(\Gamma_{0} / 10^{2.5}\right)^{-8 / 3} \mathrm{~s} & (k=0), \\
5.9 \zeta E_{53} A_{*}^{-1}\left(\Gamma_{0} / 100\right)^{-4} \mathrm{~s} & (k=2) .
\end{array}\right.
$$

Once most of the energy in the GRB outflow is transfered to the shocked external medium, the flow becomes self-similar, and is described by the Blandford \& McKee (1976) solution. The scaling of the basic quantities with radius during this stage may be understood as follows. In this stage most of the energy is in the shocked external medium, and therefore the dynamics may be found by energy conservation within this region. In the rest frame of the the shocked external medium (region 2), the cold upstream external medium approaches at a Lorentz factor $\Gamma_{12}=\Gamma_{2}$, which for simplicity we denote here by $\Gamma$, and the velocities of the particles are randomized at the shock font, such that the ordered bulk motion in the upstream region is converted to random motion of the particles in the downstream region (2), with the same average Lorentz factor. Therefore, in the rest frame of the shocked downstream medium, the average energy per particle (including its rest energy) is $\Gamma$ times its rest energy. In the lab frame the energy is larger by a factor of $\Gamma$, implying that the total kinetic energy in the lab frame is $E=\left(\Gamma^{2}-1\right) M(R) c^{2}=u^{2} M(R) c^{2}$, where $M(R)$ is the total swept-up rest mass up to radius $R, u=\Gamma \beta$ is the 4-velocity, and we have deducted the rest energy in order to obtain the kinetic energy (only the latter is conserved, since new rest-mass is added to the shocked region as the external medium is swept-up by the forward shock).

For an external density that varies as a power law with radius, $\rho_{1} \propto R^{-k}$, we have $E \propto u^{2} R^{3-k}$ and therefore conservation of energy in the lab frame 
implies that $u \propto R^{(k-3) / 2}$. For a relativistic flow $u \approx \Gamma$ and $\Gamma \propto R^{(k-3) / 2}$. Eventually the flow becomes Newtonian and approaches the Sedov-Taylor solution. In this regime $u \approx \beta$ and therefore $\beta \propto R^{(k-3) / 2}$. Both scalings given above apply to the adiabatic case where energy losses (e.g., due to radiation) or gains (e.g., due to late time energy injection from the central source) can be neglected. For simplicity we consider here only $k<3$ for which $u$ decreases with $R$. The pressure in the shocked region scales as $p \sim \rho_{1} c^{2} u^{2} \propto R^{-3}$, while the particle rest mass density (in the comoving frame) scales as $\rho_{2} \sim \Gamma \rho_{1}$, i.e., as $R^{-(3+k) / 2}$ in the relativistic case and as $R^{-k}$ for the Newtonian case. A spherical flow becomes non-relativistic once $\Gamma^{2} \approx E / M(R) c^{2} \sim 1$, at

$$
t_{\mathrm{NR}} \sim \frac{l}{c}=\left[\frac{(3-k) E}{4 \pi A c^{5-k}}\right]^{1 /(3-k)}=\left\{\begin{array}{cc}
5.3 \zeta E_{53}^{1 / 3} n_{0}^{-1 / 3} \mathrm{yr} & (k=0), \\
37 \zeta E_{53} A_{*}^{-1} \mathrm{yr} & (k=2) .
\end{array}\right.
$$

Variations on the relatively simple dynamics described above after the deceleration epoch involve relaxing either the assumption of a constant energy or of spherical symmetry. The energy can decrease due to radiative losses or increase due to energy injection, either from a relativistic wind caused by prolonged activity of the central source, or slow material that was ejected promptly from the source but catches up with the shocked external medium as the latter decelerates to a somewhat smaller Lorentz factor (e.g., Blandford \& McKee 1976, Cohen \& Piran 1999, Sari \& Mészáros 2000, Ramirez-Ruiz et al. 2001). Here we discuss deviation from spherical symmetry, for which the dynamics are more complicated and less certain. For simplicity, we shall consider an axisymmetric double-sided jet that is uniform within an initial half-opening angle of $\theta_{0} \ll 1$ around its symmetry axis with sharp edges.

If a signal (e.g., a sound or rarefaction wave) can travel laterally within the jet at a speed of $\beta_{\mathrm{s}} c$ in its comoving frame, then it traverses an angle of $d \theta \approx d R_{\perp} / R=\beta_{\mathrm{s}} c d t^{\prime} / R \approx \beta_{\mathrm{s}} d R / R \Gamma$, so that even for $\beta_{\mathrm{s}} \sim 1$ (which may indeed be expected) information traverses an angle of $\sim 1 / \Gamma$ on the dynamical (or radius doubling) time. Therefore, the center of the jet knows about its edge only when $\Gamma$ drops to $\sim 1 / \theta_{0}$; at earlier times it behaves as if it were part of a spherical flow with the same external density profile and isotropic equivalent energy $E_{\text {iso }}$. Using the spherical adiabatic scaling $\Gamma \propto R^{(k-3) / 2}$ a signal starting at $R_{0}$ traverses $\Delta \theta \approx 2 \beta_{\mathrm{s}} /(3-k) \Gamma(R)$ by a radius $R \gg R_{0}$ for $k<3$. Therefore, when $\Delta \theta=\theta_{0}$ corresponding to $\Gamma \theta_{0} \approx 2 \beta_{\mathrm{s}} /(3-k)$, the center of the jet comes into causal contact with its edge, and the jet can in principle start to spread sideways rapidly. 
Early analytic works (Rhoads 1997, 1999, Sari et al. 1999) assumed that the jet half-opening angle grows as $\theta_{\mathrm{j}}=\theta_{0}+\Delta \theta \sim \theta_{0}+\beta_{\mathrm{s}} / \Gamma$ and indeed quickly expands laterally once $\Gamma$ drops below $\sim \beta_{\mathrm{s}} / \theta_{0} \sim 1 / \theta_{0}$, around the same time as the edges of the jet become visible $t$. As a result, a steepening in the afterglow light curve is produced, which is known as a jet break and was detected soon after it was predicted. The jet break occurs at a radius $R_{\mathrm{j}} \sim$ $R_{\mathrm{NR}}(E) \sim \theta_{0}^{2 /(3-k)} R_{\mathrm{NR}}\left(E_{\mathrm{iso}}\right)$, and the simple analytic models (e.g., Rhoads $1999)$ suggest that at $R>R_{\mathrm{j}}, \Gamma \sim \theta_{0}^{-1} e^{1-R / R_{\mathrm{j}}}$ and $\theta_{\mathrm{j}} \sim \theta_{0}\left(R_{\mathrm{j}} / R\right) e^{1-R / R_{\mathrm{j}}}$ until $\Gamma \sim 1$ at $R \sim R_{\mathrm{j}}\left(1-\ln \theta_{0}\right)$. Different analytic models vary in their exact assumptions and results (e.g., Piran 2000, Granot 2007), but the exponential decrease in $\Gamma$ and increase in $\theta_{\mathrm{j}}$ with radius are a generic prediction. In this picture the jet becomes sub-relativistic at close to $R_{\mathrm{NR}}(E)$ (to within a logarithmic factor), and therefore the transition to the late time asymptotic spherical Newtonian Sedov-Taylor solution is relatively prompt and smooth in this scenario.

However, numerical studies (Granot et al. 2001, Kumar \& Granot 2003, Cannizzo et al. 2004, Zhang \& MacFadyen 2009), and in particular full special relativistic two dimensional hydrodynamic simulations, which are the best and most reliable calculations to date, show that the lateral expansion of the jet is very modest as long as it remains relativistic. The big difference compared to the results of simple analytic models may be attributed to the over-simplified assumptions, such as the jet being perfectly uniform with sharp edges within some finite half-opening angle with a purely radial velocity, which are shown to be invalid in the numerical simulations. Because of the lack of significant sideways expansion during the relativistic stage, the gross properties of the jet may be approximated to zeroth order by a conical section of half opening angle $\sim \theta_{0}$ out of a spherical flow. Therefore, the jet becomes non-relativistic at a radius $\sim R_{\mathrm{NR}}\left(E_{\text {iso }}\right) \sim \theta_{0}^{-2 /(3-k)} R_{\mathrm{NR}}(E)$, i.e., near the Sedov length corresponding to its initial isotropic equivalent energy $E_{\text {iso }}$, which for a narrow jet $\left(\theta_{0} \ll 1\right)$ is significantly larger than the Sedov length corresponding to its true energy $E \approx E_{\text {iso }} \theta_{0}^{2} / 2$. This causes the transition into a spherical flow described by the Sedov-Taylor solution (with the true energy $E$ ) to extend over a large range of times, with a rather modest growth in the (maximal) radius during this transition period (Granot et al. 2005).

$\dagger$ Since the visible region is within an angle of $\sim 1 / \Gamma$ around the line of sight due to relativistic beaming and light travel effects. 


\subsection{The afterglow emission}

The dominant emission mechanism during the afterglow stage is believed to be synchrotron radiation, produced as relativistic electrons accelerated by the afterglow shock gyrate in the magnetic fields within the shocked external medium. A synchrotron origin of the afterglow emission is supported by the detection of linear polarization at the level of $\sim 1 \%-3 \%$ in several optical or NIR afterglows (see Sect. 11.9), and by the shape of the broad band spectrum, which consists of several power-law segments that smoothly join at some typical break frequencies (Galama et al. 1998). Synchrotron self-Compton (SSC) - the inverse-Compton scattering of the synchrotron photons to (much) higher energies by the same population of relativistic electrons that emits the synchrotron photons - can sometimes dominate the afterglow flux in the X-rays (Sari \& Esin 2001, Harrison et al. 2001), and may affect the synchrotron emission by increasing the electron cooling.

Since the physics of relativistic collisionless shocks (and in particular how they amplify the magnetic field and accelerate particles to a non-thermal relativistic energy distribution) are still not well understood from first principles, simple assumptions are usually made that conveniently parameterize our ignorance. The electrons are usually assumed to be (practically instantaneously) shock-accelerated into a power-law distribution of energies, $d N / d \gamma_{e} \propto \gamma_{e}^{-p}$ for $\gamma_{e}>\gamma_{m}$, and thereafter to cool both adiabatically and due to radiative losses. It is further assumed that practically all of the electrons take part in this acceleration process and form such a non-thermal (power-law) distribution, leaving no thermal component (which is not at all clear or justified; e.g., Eichler \& Waxman 2005). The relativistic electrons are assumed to hold a fraction $\epsilon_{e}$ of the internal energy immediately behind the shock, while the magnetic field is assumed to hold a fraction $\epsilon_{B}$ of the internal energy everywhere in the shocked region.

The spectral emissivity in the comoving frame of the emitting shocked material is typically approximated as a broken power law (in some cases the more accurate functional form of the synchrotron emission is used, e.g., Wijers \& Galama 1999, Granot \& Sari 2002). Most calculations of the light curve assume emission from an infinitely thin shell, which represents the shock front (some integrate over the volume of the shocked fluid taking into account the appropriate radial profile of the flow, e.g., Granot \& Sari 2002; see Figure 11.3). One also needs to account for the different arrival times of photons to the observer from emission at different lab frame times and locations relative to the line of sight, as well as the relevant Lorentz transformations of the emission into the observer frame. SSC is included 


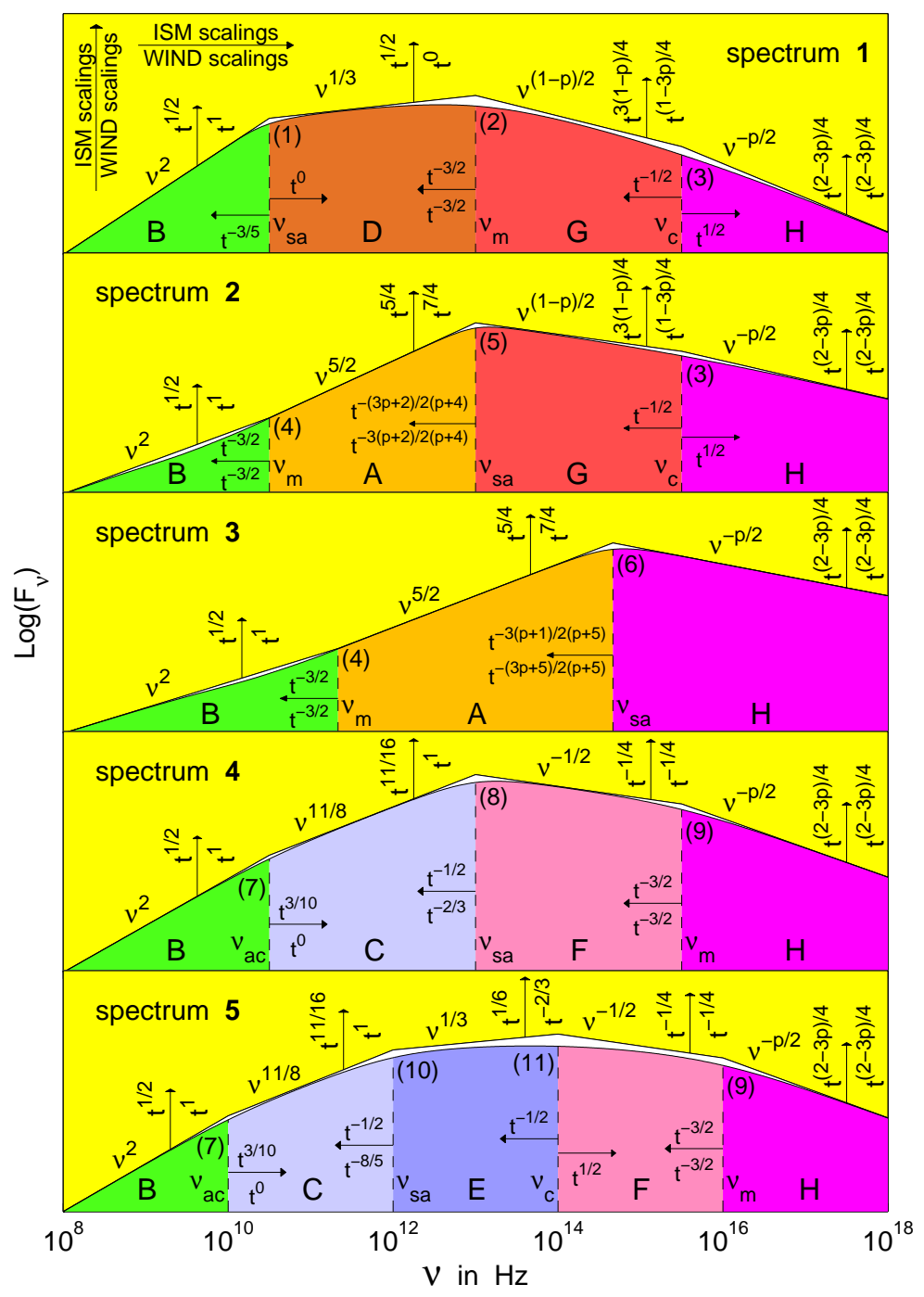

Fig. 11.3. The afterglow synchrotron spectrum, calculated for the Blandford \& McKee (1976) spherical self-similar solution, under standard assumptions, using the accurate form of the synchrotron spectral emissivity and integration over the emission from the whole volume of shocked material behind the forward (afterglow) shock (for details see Granot \& Sari 2002). The different panels show the five possible broad band spectra of the afterglow synchrotron emission, each corresponding to a different ordering of the spectral break frequencies. Each spectrum consists of several power-law segments (PLSs; each shown with a different color and labeled by a different letter $\mathrm{A}-\mathrm{H}$ ) that smoothly join at the break frequencies (numbered 1-11). The broken power-law spectrum, which consists of the asymptotic PLSs that abruptly join at the break frequencies (and is widely used in the literature), is shown for comparison. Most PLSs appear in more than one of the five different broad band spectra. Indicated next to the arrows are the temporal scaling of the break frequencies and the flux density at the different PLSs, for a uniform (ISM; $k=0$ ) and stellar wind (WIND; $k=2$ ) external density profile. 
in some (but not all) works, even though it can also effect the synchrotron emission through the enhanced radiative cooling of the electrons.

\subsection{Afterglow images}

In order to calculate the afterglow image, or how the afterglow would appear on the plane of the sky if it were angularly resolved, we need to specify the dynamics, in addition to an emission model, here assumed to be synchrotron radiation. As discussed in Sect.11.5, at early times before the jet break time in the afterglow light curve the dynamics may be reasonably approximated as part of a spherical flow that is described by the self-similar Blandford \& McKee (1976) solution. Here we shall concentrate on this stage, for which the afterglow image is also self-similar.

During the self-similar spherical evolution stage (before the jet break time, for a jet), the afterglow image has circular symmetry around the line of sight (where the surface brightness depends only on the distance from the center of the image), and is confined within a circle on the sky with a radius

$$
\frac{R_{\perp}}{10^{16} \mathrm{~cm}}= \begin{cases}3.91\left(\frac{E_{52}}{n_{0}}\right)^{1 / 8}\left(\frac{t_{\text {days }}}{1+z}\right)^{5 / 8} & (k=0), \\ 2.39\left(\frac{E_{52}}{A_{*}}\right)^{1 / 4}\left(\frac{t_{\text {days }}}{1+z}\right)^{3 / 4} & (k=2),\end{cases}
$$

(see Figure 11.4).

This corresponds to an angular radius of

$$
\frac{R_{\perp}}{d_{A}}= \begin{cases}\frac{1.61 \mu \mathrm{as}}{d_{A, 27.7}}\left(\frac{E_{52}}{n_{0}}\right)^{1 / 8}\left(\frac{t_{\mathrm{days}}}{1+z}\right)^{5 / 8} & (k=0), \\ \frac{0.98 \mu \mathrm{as}}{d_{A, 27.7}}\left(\frac{E_{52}}{A_{*}}\right)^{1 / 4}\left(\frac{t_{\mathrm{days}}}{1+z}\right)^{3 / 4} & (k=2),\end{cases}
$$

where $d_{A}(z)=10^{27.7} d_{A, 27.7} \mathrm{~cm}$ 田

More generally, the afterglow image size during the self-similar spherical stage scales with the observed time as $R_{\perp} \propto t^{(5-k) / 2(4-k)}$. The image size grows super-luminally with an apparent expansion velocity of $\Gamma_{\mathrm{sh}}\left(R_{*}\right) c$. The expected afterglow images during this self-similar regime are shown in Figures 11.5 and 11.6. The normalized surface brightness profile within the afterglow image is independent of time due to the self-similar dynamics, and changes only between the different power-law segments of the synchrotron spectrum, and for different external density profiles. The image becomes increasingly limb-brightened at higher frequencies, and for smaller values of $k$ (Granot \& Loeb 2001, Granot 2008).

$\dagger$ For a standard cosmology $\left(\Omega_{M}=0.27, \Omega_{\Lambda}=0.73, h=0.71\right) d_{A}(z)$ has a maximum value of $5.45 \times 10^{27} \mathrm{~cm}\left(d_{A, 27.7}=1.09\right)$ for $z=1.64$. 


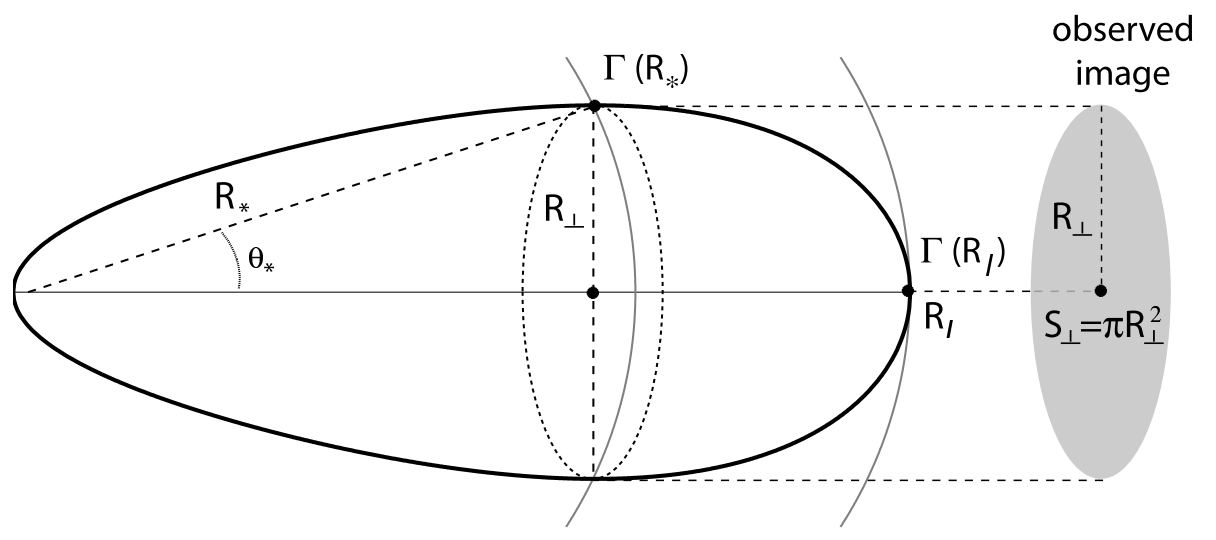

Fig. 11.4. Schematic illustration of the equal arrival time surface (thick black line), namely, the surface from where the photons emitted at the shock front arrive at the same time at the observer (on the far right-hand side). The maximal lateral extent of the observed image, $R_{\perp}$, is located at an angle, where the shock radius and Lorentz factor are $R_{*}$ and $\Gamma_{*}=\Gamma_{\operatorname{sh}}\left(R_{*}\right)$, respectively. The area of the image on the plane of the sky is $S_{\perp}=\pi R_{\perp}^{2}$. The shock Lorentz factor, $\Gamma_{\mathrm{sh}}$, varies with radius $R$ and angle $\theta$ from the line of sight along the equal arrival time surface. The maximal radius $R_{l}$ on the equal arrival time surface is located along the line of sight. If, as expected, $\Gamma_{\text {sh }}$ decreases with $R$, then $\Gamma_{l}=\Gamma_{\text {sh }}\left(R_{l}\right)$ is the minimal shock Lorentz factor on the equal arrival time surface. (from Granot et al. 2005).

Below the self-absorption frequency, the specific intensity (surface brightness) represents the Rayleigh-Jeans portion of a black-body spectrum with the blue-shifted effective temperature of the electrons at the corresponding radius along the front side of the equal arrival time surface of photons to the observer $\left(R_{*} \leq R \leq R_{l}\right.$ in Figure 11.4). Above the cooling break frequency the emission originates from a very thin layer behind the shock front, where the electrons whose typical synchrotron frequency is close to the observed frequency have not yet had enough time to significantly cool due to radiative losses. This results in a divergence of the surface brightness at the outer edge of the image (Sari 1998, Granot \& Loeb 2001).

After the jet break time the afterglow image is no longer symmetric around the line of sight to the central source for a general viewing angle (which is not exactly along the jet symmetry axis), and its details depend on the hydrodynamic evolution of the jet (so that in principle it could be used to constrain the jet dynamics). Therefore, a realistic calculation of the afterglow image during the more complicated post-jet break stage requires the use of hydrodynamic simulations, and still remains to be done.

The afterglow image may be indirectly resolved via gravitational lensing 


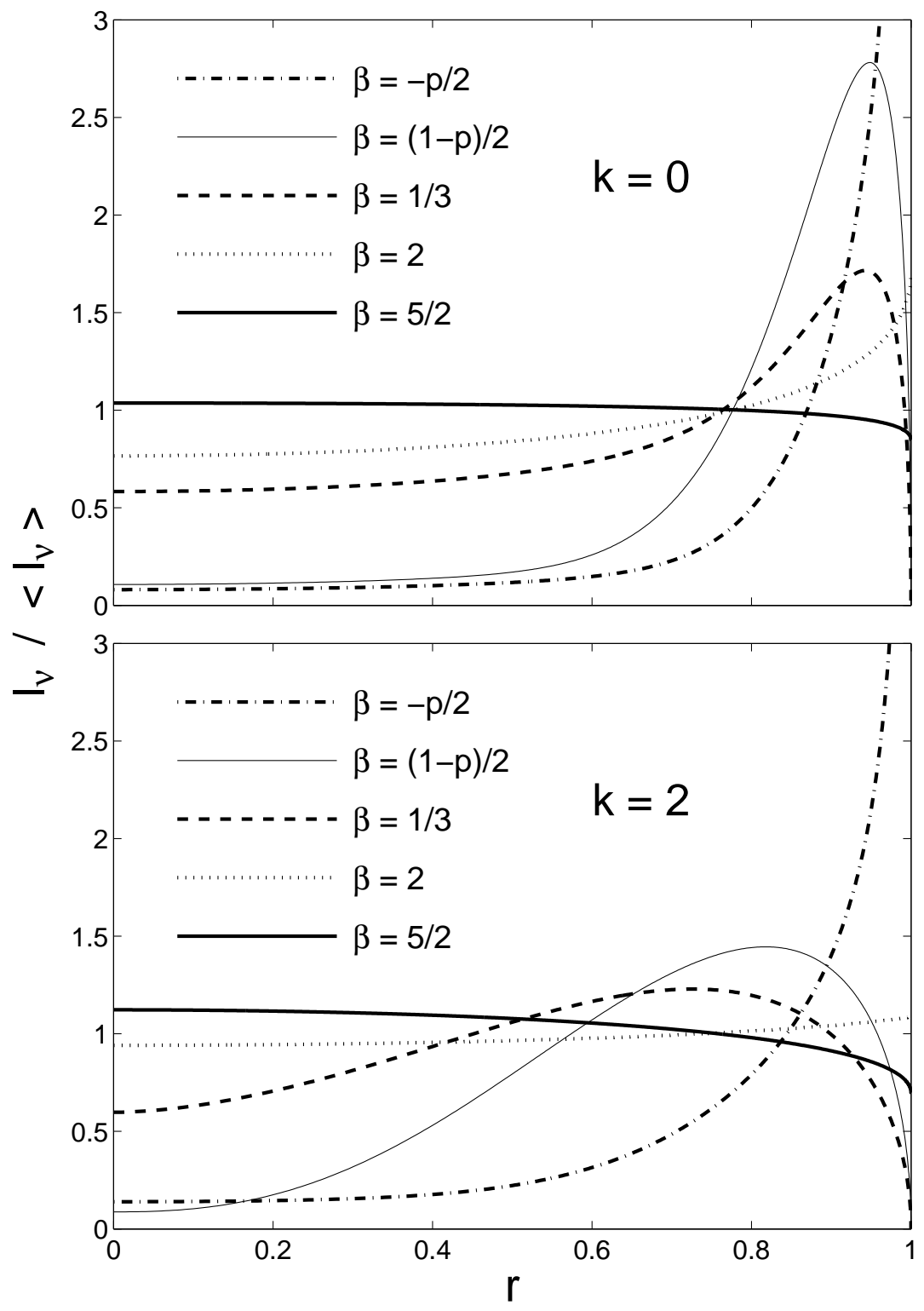

Fig. 11.5. The afterglow images for different power-law segments of the spectrum, for a uniform $(k=0)$ and wind $(k=2)$ external density profile (from Granot \& Loeb 2001), calculated for the Blandford \& McKee (1976) spherical self similar solution, using the formalism of Granot \& Sari (2002). Shown is the surface brightness, normalized by its average value, as a function of the normalized distance from the center of the image, $r=R \sin \theta / R_{\perp}$ (where $r=0$ at the center and $r=1$ at the outer edge). The image profile changes considerably between different power-law segments of the afterglow spectrum, $F_{\nu} \propto \nu^{\beta}$. There is also a strong dependence on the density profile of the external medium, $\rho_{\text {ext }} \propto R^{-k}$. 

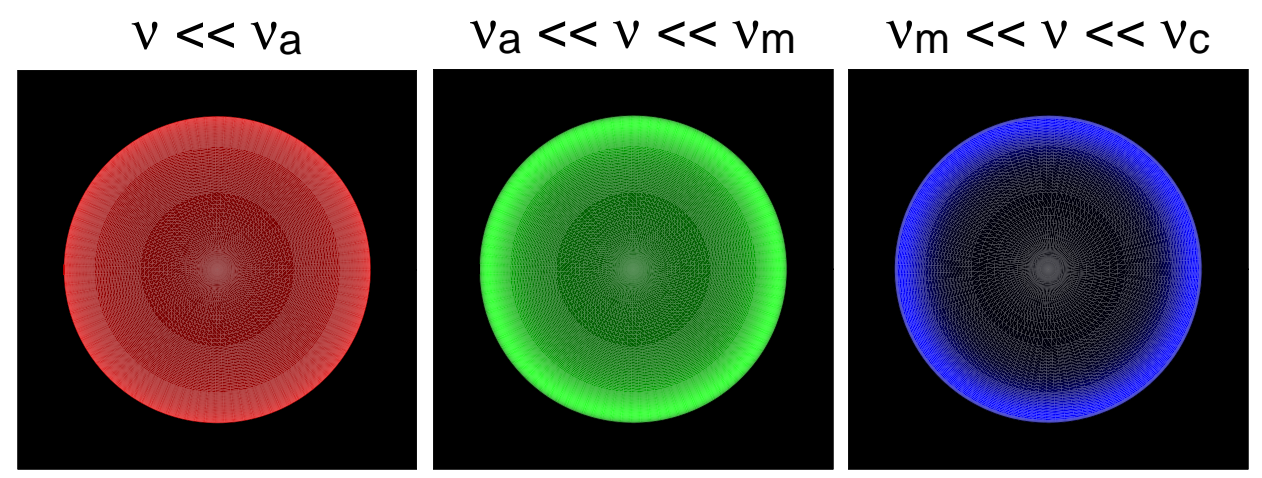

Fig. 11.6. An illustration of the expected afterglow image on the plane of the sky, for three different power-law segments of the spectrum (from Granot et al. 1999a,b), assuming a uniform external density and the Blandford McKee (1976) self-similar solution. The image is more limb brightened at power-law segments that correspond to higher frequencies.

by a star in an intervening galaxy (along, or close to, our line of sight to the source). This is because the angular size of the Einstein radius (i.e., the region of large magnification around the lensing star) for a typical star at a cosmological distance is $\sim 1 \mu$ as (hence the name micro-lensing) - comparable to the afterglow image size after a day or so. Since the afterglow image size grows very rapidly with time, different parts of the image sample the regions of large magnification (close to the point of infinite magnification just behind the lensing star) with time, and therefore the overall magnification of the afterglow flux as a function of time probes the surface brightness profile of the afterglow image. This results in a bump in the afterglow light curve, which peaks when the limb-brightened outer part of the image sweeps past the lensing star; the peak of the bump is sharper when the afterglow image is more limb-brightened (Granot \& Loeb 2001). It has been suggested that an achromatic bump in the afterglow light curve of GRB 000301C after $\sim 4$ days might have been due to micro-lensing (Garnavich et al. 2000). If this interpretation is true, then the shape of the bump in the afterglow light curve requires a limb-brightened afterglow image, in agreement with theoretical expectations (Gaudi et al. 2001).

\subsection{The cause of the jet break}

The jet break in the afterglow light curve has been argued to be the combination of (i) the edge of the jet becoming visible, and (ii) fast lateral spreading. Both effects are expected to take place around the same time, 
when the Lorentz factor, $\Gamma$, of the jet drops below the inverse of its initial half-opening angle, $\theta_{0}$. This can be understood as follows.

When $\Gamma$ drops below $\theta_{0}^{-1}$, the edge of the jet becomes visible. This is because relativistic beaming limits the region from which a significant fraction of the emitted radiation reaches the observer to within an angle of $\sim \Gamma^{-1}$ around the line of sight $\left(\theta \lesssim \Gamma^{-1}\right)$. Once the edge of the jet becomes visible, then, if there is no significant lateral spreading, only a small fraction, $\left(\Gamma \theta_{\mathrm{j}}\right)^{2}<1$, of the visible region is occupied by the jet and, as a result, there would be "missing" contributions to the observed flux compared to a spherical flow. This would cause a steepening in the light curve, i.e., a jet break, where the temporal decay index asymptotically increases by $\Delta \alpha=(3-k) /(4-k)$ (since the fraction of the visible region occupied by the jet is $\left.\left(\Gamma \theta_{\mathrm{j}}\right)^{2} \sim\left(t / t_{\mathrm{j}}\right)^{-(3-k) /(k-4)}\right)$.

When $\Gamma$ drops below $\theta_{0}^{-1}$, the center of the jet comes into causal contact with its edge, and the jet can in principle start to expand sideways significantly. It has been argued that at this stage it would indeed start to expand sideways rapidly, at close to the speed of light in its own rest frame. In this case, during the rapid lateral expansion phase the jet opening angle grows as $\theta_{\mathrm{j}} \sim \Gamma^{-1}$ and exponentially with radius (see Sect. 11.5). This causes the energy per unit solid angle, $\mathcal{E}$, in the jet to drop with observed time, and the Lorentz factor to decrease faster as a function of observed time; this gives rise to a steepening in the afterglow light curve compared to a spherical flow (for which $\mathcal{E}$ remains constant and $\Gamma$ decreases more slowly with the observed time). However, in this case a large fraction of the visible region remains occupied by the jet (since $\Gamma \theta_{\mathrm{j}}$ remains $\sim 1$ ), so that the first cause for the jet break (the edge of the jet becoming visible, and the "missing" contributions from outside the edge of the jet) is no longer important. Therefore, for fast lateral spreading, the jet break occurs predominantly both as a result of the energy per solid angle $\mathcal{E}$ decreasing with time and the Lorentz factor decreasing with observed time faster than for a spherical flow.

It is important to keep in mind, however, that numerical studies show that the lateral spreading of the jet is very modest as long as it is relativistic (see Sect. (11.5). This implies that lateral spreading cannot play an important role in the jet break, and the predominant cause of the jet break is the "missing" contribution from outside of the jet, once its edge becomes visible.

A potential problem with this description is that if the jet half-opening angle remains roughly constant, $\theta_{\mathrm{j}} \approx \theta_{0}$, the asymptotic change in the temporal decay index is only $\Delta \alpha=3 / 4$ for a uniform external medium $(k=0)$ or even smaller for a wind $(\Delta \alpha=1 / 2$ for $k=2)$, while the values inferred from observations are in most cases larger (see Figure 3 of Zeh et al. 2006). 

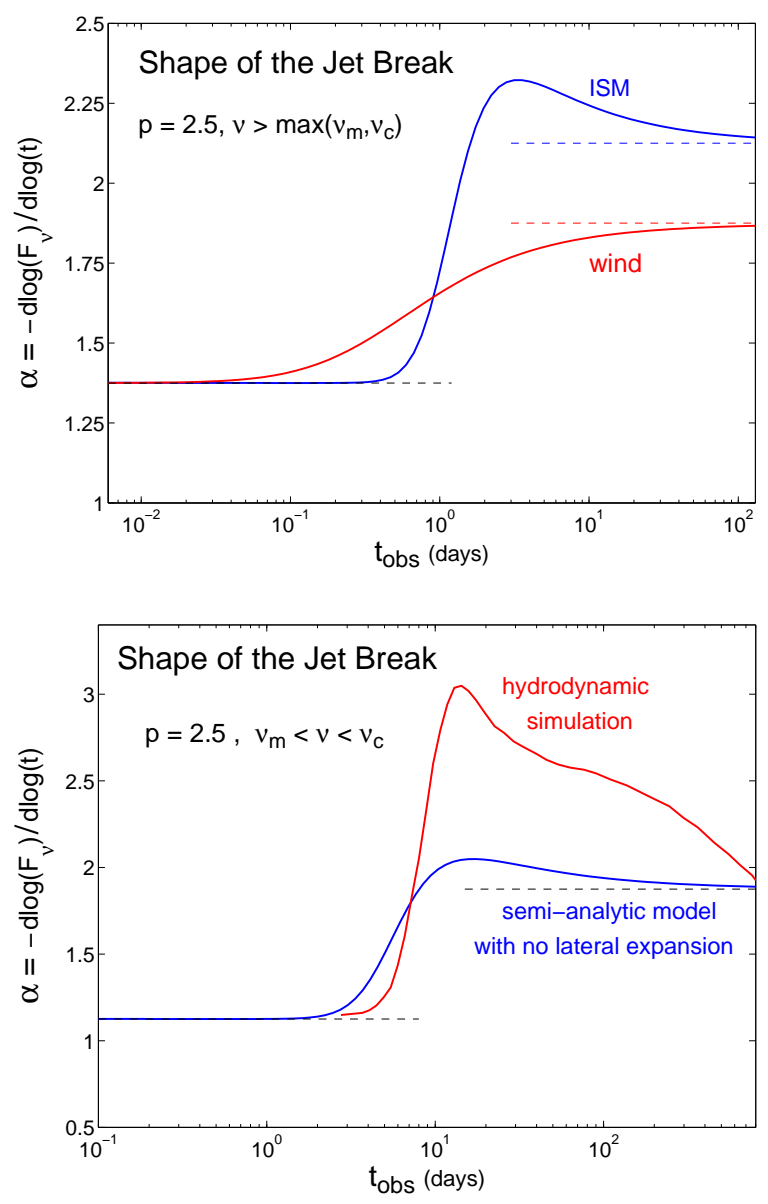

Fig. 11.7. The temporal decay index $\alpha$ as a function of the observed time (in days) across the jet break in the light curve, for $p=2.5$. Top panel: results in the spectral range $\nu>\max \left(\nu_{m}, \nu_{c}\right)$ using a semi-analytic model with no lateral spreading (Granot 2005), for a uniform $\left(k=0, n_{\text {ext }}=1 \mathrm{~cm}^{-3}\right)$ and wind $\left(k=2, A_{*}=1\right)$ external density profile, with $\theta_{0}=0.1$ and $E_{\mathrm{k} \text {,iso }}=2 \times 10^{53} \mathrm{erg}$. Bottom panel: results for the spectral range $\nu_{m}<\nu<\nu_{c}$, for $\theta_{0}=0.2$ and a uniform density $(k=0$, $\left.n_{\text {ext }}=1 \mathrm{~cm}^{-3}, E_{\mathrm{k} \text {,iso }}=10^{52} \mathrm{erg}\right)$; the figure compares the result of a semi-analytic model (Granot 2005) to those of a hydrodynamic simulation (Granot et al. 2001). In both panels the dashed lines show the asymptotic values of $\alpha$ before and after the jet break, for a uniform jet with no lateral spreading, for which $\Delta \alpha=(3-k) /(4-k)$.

This apparent discrepancy may be reconciled as follows. While the asymptotic steepening is indeed $\Delta \alpha=(3-k) /(4-k)$ when lateral expansion is negligible, the value of the temporal decay index $\alpha$ (where $F_{\nu} \propto t^{-\alpha}$ ) initially overshoots its asymptotic value. Since the temporal baseline that is 
used to estimate the post-jet break temporal decay index $\alpha_{2}$ is typically no more than a factor of several in time after the jet break timet $t_{\mathrm{j}}$, the value of $\alpha$ during this time is larger than its asymptotic value, $\alpha_{2}$. This causes the value of $\Delta \alpha$ that is inferred from observations to be larger than its true asymptotic value.

The overshoot in the value of $\alpha$ just after the jet break time can be seen in Figure 11.7 and is much more pronounced in the light curves calculated using the jet dynamics from a hydrodynamic simulation, compared to the results of a simple semi-analytic model. This overshoot occurs because the afterglow image is limb-brightened (see Figure 11.5) and therefore its brightest part - its outer edges - is the first region whose contribution to the observed flux is "missed" as the edge of the jet becomes visible. The overshoot increases for increasingly limb-brightened afterglow images (e.g.,, for $\nu>\max \left[\nu_{m}, \nu_{c}\right]$ in the upper panel of Figure 11.7 compared to $\nu_{m}<\nu<\nu_{c}$ in the lower panel of Figure 11.7). For a wind density $(k=2)$ the limbbrightening is smaller compared to a uniform density $(k=0)$, at the same power-law segment of the spectrum (see Figure 11.5), and the Lorentz factor $\Gamma$ decreases more slowly with the observed time. Because of this no overshoot is seen in the semi-analytic model shown in the upper panel of Figure 11.7 for a wind density profile $(k=2)$, and the jet break is smoother and extends over a larger factor in time. The asymptotic post-jet break value of the temporal decay index $\left(\alpha_{2}\right)$ is approached only when the part of the image of the corresponding spherical flow that is occupied by the jet covers only the relatively uniform central part of the image, and not its brighter outer edge.

The jet break in light curves calculated from hydrodynamic simulations is sharper than in semi-analytic models (where the emission is taken to be from a 2D surface - usually a section of a sphere within a cone). In semi-analytic models the jet break is sharpest with no lateral expansion, and becomes more gradual with faster assumed lateral expansion. For example, in the lower panel of Figure 11.7, where the viewing angle is along the jet axis and the external density is uniform, most of the change in the temporal decay index $\alpha$ occurs over a factor of $\sim 2$ in time for the numerical simulation, and over a factor of $\sim 3$ in time for the semi-analytic model (which assumes no lateral expansion; the jet break would be more gradual with lateral expansion). For both types of models, the jet break is more gradual and occurs at a somewhat later time for viewing angles further away from the jet symmetry axis but still within its initial opening angle, although this effect is somewhat

$\dagger$ This is usually because the flux becomes too dim to detect above the host galaxy, or since a supernova component becomes dominant in the optical. 
more pronounced in semi-analytic models (Granot et al. 2001, Rossi et al. 2004).

\subsection{Polarization: afterglow and prompt}

Afterglow: Linear polarization at the level of a few percent has been detected in the optical and NIR afterglow of several GRBs (see Covino et al. 2004 and references therein). This was considered to be a confirmation that synchrotron radiation is the dominant emission mechanism in the afterglow. The synchrotron emission from a fluid element with a locally uniform magnetic field is linearly polarized in the direction perpendicular to the projection of the magnetic field onto the plane normal to the wave vector. Since the source moves relativistically, aberration of light (see Sect. 11.2) must be accounted for when calculating the observed local direction of polarization. Figure 11.8 shows the predicted local polarization map from emission by an ultra-relativistic expanding shell, for two different magnetic field structures: a magnetic field that is random within the plane normal to the radial direction (left panel) as could be expected from a shock produced magnetic field (e.g., Medvedev \& Loeb 1999), and an ordered magnetic field normal to the radial direction (as could be expected in the prompt emission for a magnetic field coherent on angular scales $\gg 1 / \Gamma_{0}$ that is advected from the central source).

Since the afterglow image is almost always never resolved, we can only measure the average polarization over the whole image. For this reason, a shock produced magnetic field that is symmetric about the shock normal will procure no net polarization for a spherical flow (since the polarization pattern across the image is in this case symmetric around the center of the image, and the polarization averages out to zero when summing over the contributions from the whole image). For a shock-produced magnetic field one thus needs to break the spherical symmetry of the flow to produce net polarization. The simplest and most natural way of doing this is considering a jet, or narrowly collimated outflow (e.g., Sari 1999, Ghisellini \& Lazzati 1999). In this picture a jet geometry together with a line of sight that is not along the jet symmetry axis (but still within the jet aperture, in order to see the prompt GRB) is needed to break the symmetry of the afterglow image around our line of sight.

For a uniform jet (the UJ model) this predicts two peaks in the polarization light curve around the jet break time $t_{\mathrm{j}}$, if $\Gamma \theta_{\mathrm{j}}<1$ decreases with time at $t>t_{\mathrm{j}}$ (Ghisellini \& Lazzati 1999, Rossi et al. 2004), or even three peaks if $\Gamma \theta_{\mathrm{j}} \approx 1$ at $t>t_{\mathrm{j}}$ (Sari 1999), where in both cases the polariza- 

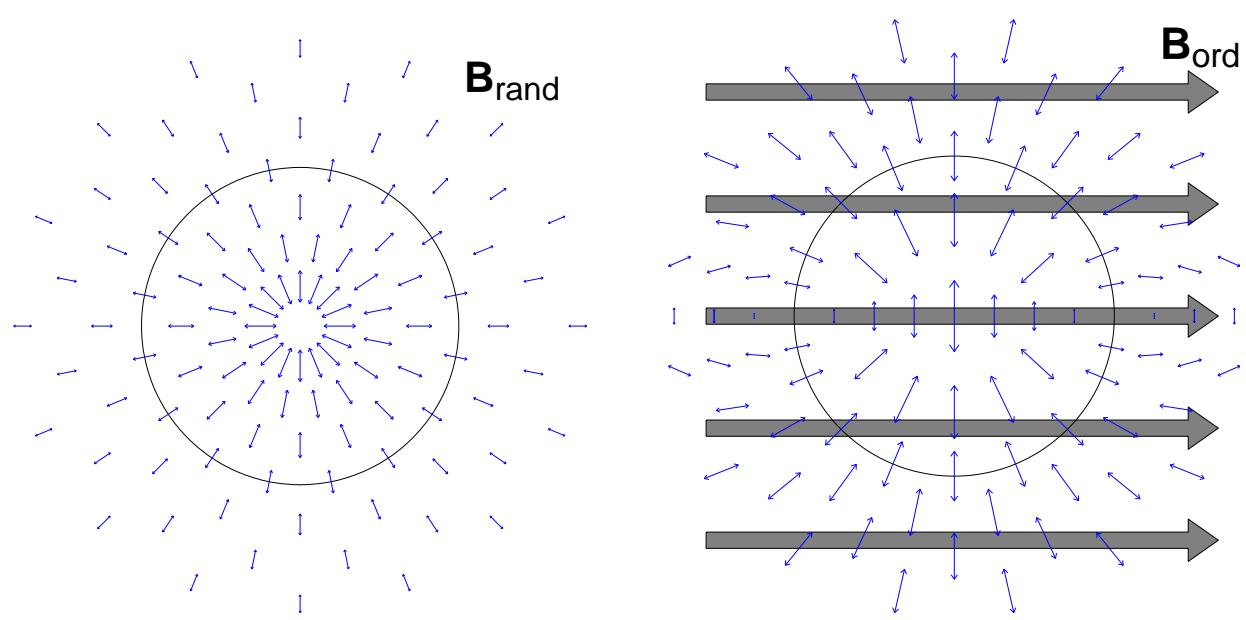

Fig. 11.8. The predicted polarization map for synchrotron emission from a thin spherical ultra-relativistic shell expanding with a Lorentz factor $\Gamma \gg 1$. The doublesided arrows show the direction of the linear polarization (the wave electric vector), while their length depends monotonically on the polarized intensity (in a non-trivial way, for display purposes). The circle indicates an angle of $1 / \Gamma$ around the line of sight, which is the region responsible for most of the observed flux. The left panel is for a magnetic field that is random within the plane of the shell (normal to the radial direction), for which the polarization direction always points at the center of the image (corresponding to the line of sight to the center of the spherical shell), where the polarization vanishes (due to symmetry consideration). The right panel is for an ordered magnetic field within the plane of the shell that is coherent over angular scales $\gg 1 / \Gamma$ (Granot \& Königl 2003). In this case the direction of the ordered magnetic field clearly breaks the symmetry around the center of the image, resulting in a large net polarization. For simplicity, the map is for a constant emission radius, rather than for a constant photon arrival time.

tion vanishes and reappears rotated by $90^{\circ}$ between adjacent peaks. The latter is a distinct signature of this model. For a structured jet (the USJ model), the polarization position angle is expected to remain constant in time, while the degree of polarization peaks near the jet break time $t_{\mathrm{j}}$ (Rossi et al. 2004). A similar qualitative behavior is also expected for a Gaussian jet, or other jet structures with a bright core and dimmer wings (although there are obviously some quantitative differences).

The different predictions for the afterglow polarization light curves for different jet structures imply that afterglow polarization observations could in principle help constrain the jet structure. In practice, however, the situation is much more complicated, mainly since the observed polarization depends not only on the jet geometry, but also on the magnetic field configuration within the emitting region, which is not well known. For example, an or- 
dered magnetic field component in the emitting region (e.g., due to a small ordered magnetic field in the external medium) may dominate the polarized flux, and therefore the polarization light curves, even if it is sub-dominant in the emitting region compared to a random (shock generated) magnetic field component in terms of the total energy in the magnetic field (Granot \& Königl 2003). Other models for afterglow polarization include a magnetic field that is coherent over patches of a size comparable to that of causally connected regions (Gruzinov \& Waxman 1999), and polarization that is induced by microlensing (Loeb \& Perna 1998) or by scintillations in the radio (Medvedev \& Loeb 1999).

The last two models involve mechanisms that give a different weight to emission from different parts of the afterglow image, and thus break its symmetry around the line of sight for a shock produced magnetic field (so that the polarization from the different parts of the image no longer fully cancels out). While microlensing or radio scintillations are external to the source, there are also mechanisms intrinsic to the source that can produce a similar effect on the polarization. An example is a "patchy shell", where the energy per solid angle and the corresponding afterglow brightness vary randomly with the lateral location within the flow (see Sect. 11.4). As the afterglow shock decelerates and the angular size of the visible region (of $\lesssim \Gamma^{-1}$ around the line of sight) increases, new bright and dim regions become visible and cause correlated variability in the total afterglow flux (bumps or wiggles in the afterglow light curve) and in its linear polarization - both in the degree of polarization and its position angle (Nakar \& Oren 2004). Soon after this was predicted (Granot \& Königl 2003) $\dagger$ such a correlated variability in the afterglow light curve and its polarization was reported for two particularly variable optical afterglows, of GRB 021004 (Rol et al. 2003) and GRB 030329 (Greiner et al. 2003).

Prompt Emission: in the prompt soft gamma-ray emission the nature of the dominant emission mechanism remains uncertain. Moreover, it is very difficult to measure the polarization at such photon energies (hard X-rays or soft gamma-rays), compared to the optical, NIR, or radio. There have been some claims (Coburn \& Boggs 2003, Willis et al. 2005, McGlynn et al. 2007, Götz et al. 2009) of detection of a high degree of linear polarization in the prompt gamma-ray emission of some GRBs (with a particularly high fluence, as good photon statistics are crucial for such measurements). However, these claims remain rather controversial (Rutledge \& Fox 2004, Wigger et al.

$\dagger$ A correlated variability in the afterglow light curve and its polarization is also expected in other scenarios, such as microlensing, radio scintillation and non-axisymmetric "refreshed shocks" or variations in the external density. 
2004). A detection of polarization during the prompt emission phase could nonetheless help constrain the dominant emission mechanism.

An ordered magnetic field within the outflow on angular scales $\gtrsim 1 / \Gamma$ can produce a high net polarization, of tens of percent (Granot \& Königl 2003, Granot 2003, Lyutikov et al. 2003), which is only slightly smaller than the maximal polarization of the local synchrotron emission, as the polarization vector is more or less aligned in the regions that contribute the most to the observed flux (see right panel of Figure 11.8). Such a high polarization can also be produced by synchrotron emission from a shock generated magnetic field (Waxman 2003, Granot 2003, Nakar et al. 2003) or bulk inverse-Compton scattering of an external photon field (Shaviv \& Dar 1995, Lazzati et al. 2004). However, these mechanisms require the line of sight to be near the edge of the jet (to within an angle of $\lesssim 1 / \Gamma$ ). $甘$ In order for such a line of sight not to be too rare, a narrow jet is required: $\theta_{0} \lesssim \mathrm{a}$ few $\Gamma^{-1}$. This requirement may be viable for the brightest GRBs, for which the prompt polarization can be measured, as such bright events usually correspond to very narrow jets. Nevertheless, statistical studies over a sample of GRBs, or time resolved polarimetry of different emission episodes within a single very bright GRB, may help distinguish between the different possible causes for polarization and teach us about the dominant emission mechanism, the jet structure, or the magnetic field configuration within the GRB outflow.

\subsection{Light curves for off-axis viewing angles}

The observed flux density $F_{\nu}=d E / d A d \nu d t$ is the energy per unit area, frequency and time in the direction $\hat{n}_{d}$ normal to a surface area $d A$ (at the detector). The differential contribution to it is $d F_{\nu}\left(\hat{n}_{d}\right)=I_{\nu}(\hat{n}) \cos \theta_{s d} d \Omega_{s d}$ where $\cos \theta_{s d}=\hat{n} \cdot \hat{n}_{d}, \hat{n}$ being the local direction from the relevant part of the source to the observer (or detector), $d \Omega_{s d}=d \phi_{s d} d \cos \theta_{s d}$ is the differential solid angle sustained by the contributing portion of the source as viewed from the observer, and $I_{\nu}(\hat{n})=d E / d A d \Omega d \nu d t$ is the specific intensity (the energy per unit area, time and frequency of radiation directed within a small solid angle $d \Omega$ centered on the direction $\hat{n}$ ). Since in practice almost always $\theta_{s d} \ll 1$, one can simplify the expression for $F_{\nu}$ by approximating $\cos \theta_{s d} \approx 1$. Furthermore, we have $d \Omega_{s d}=d S_{\perp} / d_{A}^{2}$ where $d_{A}(z)$ is the angular distance to the source, and $d S_{\perp}$ is the differential area in the plane of the sky (normal

$\dagger$ A significant change in the brightness of the jet on an angular scale of $\sim 1 / \Gamma$ around the line of sight can mimic a line of sight near the edge of a uniform jet, and produce a similarly high degree of polarization. 
to $\hat{n}$ ) sustained by the source, so that $d F_{\nu}=I_{\nu} d S_{\perp} / d_{A}^{2}$. Let us denote with a subscript $z$ quantities measured at the cosmological frame of the source. For an optically thin source $I_{\nu_{z}}=\int j_{\nu_{z}} d s_{z}$, where $j_{\nu_{z}}=d E_{z} / d V_{z} d \Omega_{z} d \nu_{z} d t_{z}$ is the emitted energy per unit volume, solid angle, frequency and time, while $d s_{z}$ is the differential path length along the trajectory of a photon that reaches the observer at the time $t_{\mathrm{obs}}$ when $F_{\nu}$ is measured. Since $I_{\nu} / \nu^{3}$, $j_{\nu} / \nu^{2}$ and $d s / \nu$ are Lorentz invariant (Rybicki \& Lightman 1979), we have $I_{\nu}=\left(\nu / \nu_{z}\right)^{3} I_{\nu_{z}}=(1+z)^{-3} \int j_{\nu_{z}} d s_{z}$. In addition, since $d_{A}=d_{L} /(1+z)^{2}$, where $d_{L}(z)$ is the luminosity distance, then $d F_{\nu}=j_{\nu_{z}} d V_{z}(1+z) / d_{L}^{2}$, where $d V_{z}=d S_{\perp} d s_{z}$ is the volume element in the source cosmological frame. Here $j_{\nu_{z}}=[\Gamma(1-\hat{n} \cdot \vec{\beta})]^{-2} j_{\nu^{\prime}}^{\prime}$ is measured in the source frame, while $j_{\nu^{\prime}}^{\prime}$ is measured in the (comoving) rest frame of the emitting material, which moves at a velocity $\vec{\beta} c$ in the source frame. Altogether, this gives

$$
F_{\nu}\left(t_{\mathrm{obs}}, \hat{n}\right)=\frac{(1+z)}{d_{L}^{2}(z)} \int d^{4} x \delta\left(t_{z}-\frac{t_{\mathrm{obs}}}{1+z}-\frac{\hat{n} \cdot \vec{r}}{c}\right) \frac{j_{\nu^{\prime}}^{\prime}}{\Gamma^{2}(1-\hat{n} \cdot \vec{\beta})^{2}},
$$

where $\nu^{\prime}=(1+z) \Gamma(1-\hat{n} \cdot \vec{\beta}) \nu$, and $t_{z}$ is the coordinate time at the source's cosmological frame. Since $d^{4} x=d t_{z} d V_{z}=d t_{z} d S_{\perp} d s_{z}=d t_{z} d S_{\perp} d s^{\prime}\left(\nu_{z} / \nu^{\prime}\right)=$ $d t_{z} d V^{\prime} / \Gamma(1-\hat{n} \cdot \vec{\beta})$ and $4 \pi j_{\nu^{\prime}}^{\prime} d V^{\prime}=d L_{\nu^{\prime}}^{\prime}=4 \pi\left(d E^{\prime} / d \Omega^{\prime} d \nu^{\prime} d t^{\prime}\right)$ is the differential of the isotropic equivalent spectral luminosity in the comoving frame, eq. 11.8 can be rewritten as

$$
F_{\nu}\left(t_{\mathrm{obs}}, \hat{n}\right)=\frac{(1+z)}{4 \pi d_{L}^{2}(z)} \int d t_{z} \delta\left(t_{z}-\frac{t_{\mathrm{obs}}}{1+z}-\frac{\hat{n} \cdot \vec{r}}{c}\right) \int \frac{d L_{\nu^{\prime}}^{\prime}}{\Gamma^{3}(1-\hat{n} \cdot \vec{\beta})^{3}} .
$$

This result can be intuitively understood as follows. For a point source of luminosity $L$ we have $F \equiv L / 4 \pi d_{L}^{2}$ by the definition of the luminosity distance, and thus $F_{\nu}=d F / d \nu=\left(d L / d \nu_{z}\right)\left(d \nu_{z} / d \nu\right) / 4 \pi d_{L}^{2}=L_{\nu_{z}}(1+z) / 4 \pi d_{L}^{2}$, where $L_{\nu_{z}}=4 \pi\left(d E_{z} / d \Omega_{z} d \nu_{z} d t_{z}\right)=\left(d E_{z} / d E^{\prime}\right)\left(d \Omega^{\prime} / d \Omega_{z}\right)\left(d \nu^{\prime} d t^{\prime} / d \nu_{z} d t_{z}\right) L_{\nu^{\prime}}^{\prime}$ $=\left(\nu_{z} / \nu^{\prime}\right)^{3} L_{\nu^{\prime}}^{\prime}=L_{\nu^{\prime}}^{\prime} / \Gamma^{3}(1-\hat{n} \cdot \vec{\beta})^{3}$ and we have used the relations $d \Omega^{\prime} / d \Omega_{z}=$ $\mathcal{D}^{2}=\left(\nu_{z} / \nu^{\prime}\right)^{2}$ and $d \nu^{\prime} d t^{\prime}=d \nu_{z} d t_{z}$ derived in Sect. 11.2, This is the basic result for a point source, and the flux from a source of finite size can be obtained by dividing it into a large number of small regions that may be treated as individual point sources (in the sense that $j_{\nu_{z}}=[\Gamma(1-\hat{n} \cdot \vec{\beta})]^{-2} j_{\nu^{\prime}}^{\prime}$ does not vary a lot within such a region) and summing over their contributions, as manifested in eq. 11.9,

In this section we are particularly interested in the dependence of the observed light curve, $F_{\nu}\left(t_{\mathrm{obs}}, \hat{n}\right)$, with viewing angle $\theta_{\text {obs }}$ relative to the symmetry axis of the jet, that points at some direction $\hat{n}_{\mathrm{j}}$, so that $\cos \theta_{\text {obs }}=$ $\hat{n} \cdot \hat{n}_{\mathrm{j}}$. For a jet that possesses such axial symmetry, the observed flux 
depends only on $\theta_{\text {obs }}$ and not on the azimuthal viewing angle $\phi_{\text {obs }}$, so that $F_{\nu}=F_{\nu}\left(t_{\mathrm{obs}}, \theta_{\mathrm{obs}}\right)$. In order to gain some intuition for how the afterglow light curve varies with viewing angle it is useful to first consider the simplest case of a point source moving at an angle of $\theta_{\text {obs }} \ll 1$ from the line of sight with $\Gamma \gg 1$ where $\Gamma \propto R^{-m / 2}$. In this case we can write

$$
\begin{gathered}
F_{\nu}\left(t_{\mathrm{obs}}, \theta_{\mathrm{obs}}\right) \approx \frac{1+z}{4 \pi d_{L}^{2}} \mathcal{D}^{3} L_{\nu^{\prime}}^{\prime}(R), \quad \mathcal{D}\left(R, \theta_{\mathrm{obs}}\right) \equiv \frac{\nu_{z}}{\nu^{\prime}} \approx \frac{2 \Gamma(R)}{1+\left[\Gamma(R) \theta_{\mathrm{obs}}\right]^{2}}, \\
t_{\mathrm{obs}}\left(R, \theta_{\mathrm{obs}}\right) \approx \frac{(1+z) R}{2 c \Gamma^{2}(R)}\left(\frac{1}{1+m}+\left[\Gamma(R) \theta_{\mathrm{obs}}\right]^{2}\right),
\end{gathered}
$$

where one uses the appropriate value of $R\left(t_{\mathrm{obs}}, \theta_{\mathrm{obs}}\right)$ according to eq. 11.11, For a given value of $\Gamma$, the Doppler factor $\mathcal{D}$ is roughly constant $(\sim \Gamma)$ for viewing angles within the "beaming cone" of half-opening angle $1 / \Gamma$ around the direction of motion (i.e., for $\theta_{\mathrm{obs}} \lesssim 1 / \Gamma$ ) and rapidly decreases as $\mathcal{D} \propto \theta_{\text {obs }}^{-2}$ for viewing angles outside the beaming cone $\left(\theta_{\text {obs }}>1 / \Gamma\right)$. It is convenient to compare the observed flux density $F_{\nu}$ at different viewing angles $\theta_{\mathrm{obs}}$ and observed times $t_{\mathrm{obs}}$, that originate from the same emission radius $R$ (Granot et al. 2002). In particular, $F_{\nu}\left(\theta_{\text {obs }}>0\right)$ with $F_{\nu}\left(\theta_{\text {obs }}=0\right)$,

$$
\begin{gathered}
F_{\nu}\left(t_{\mathrm{obs}}, \theta_{\mathrm{obs}}\right) \approx a^{3} F_{\nu / a}\left(b t_{\mathrm{obs}}, 0\right), \quad a \equiv \frac{\mathcal{D}\left(R, \theta_{\mathrm{obs}}\right)}{\mathcal{D}(R, 0)} \approx \frac{1}{1+\left[\Gamma(R) \theta_{\mathrm{obs}}\right]^{2}}, \\
b \equiv \frac{t_{\mathrm{obs}}(R, 0)}{t_{\mathrm{obs}}\left(R, \theta_{\mathrm{obs}}\right)} \approx \frac{1}{1+(1+m)\left[\Gamma(R) \theta_{\mathrm{obs}}\right]^{2}} \sim a .
\end{gathered}
$$

If the source decelerates, as expected during the afterglow $(m>0)$, then at early times $\Gamma \theta_{\text {obs }} \gg 1$ and we have $t_{\text {obs }} \approx(1+z) \theta_{\text {obs }}^{2} R / 2 c \propto R$ and $a \approx(1+m) b \approx\left[\Gamma(R) \theta_{\text {obs }}\right]^{-2} \propto R^{m} \propto t_{\text {obs }}^{m}$. For an on-axis flux density of $F_{\nu}\left(0, t_{\mathrm{obs}}\right) \propto \nu^{-\beta} t_{\mathrm{obs}}^{-\alpha}$, this implies that $F_{\nu}\left(\theta_{\mathrm{obs}}, t_{\mathrm{obs}}\right) \propto \nu^{-\beta} t_{\mathrm{obs}}^{-\alpha+m(3+\beta-\alpha)}$. If the jet is assumed to be non-expanding one can thus approximate $\Gamma(R)$ at the center of the jet by the expression for a spherical flow, for which $m=3-k$ (see Sect. 11.5), so that for a stellar wind environment $(k=2)$, the flux rises as $t_{\mathrm{obs}}^{3+\beta-2 \alpha} \sim t_{\mathrm{obs}}^{1}-t_{\mathrm{obs}}^{2}$ (for $\beta \sim 1$ and $\alpha \sim 1-1.5$ ), while for a uniform external medium $(k=0)$, the flux rises much more steeply as $t_{\mathrm{obs}}^{9+3 \beta-4 \alpha} \sim t_{\mathrm{obs}}^{6}-t_{\mathrm{obs}}^{8}$. At late times when the beaming cone widens enough and engulfs the line of sight $\left(\Gamma \theta_{\text {obs }}<1\right)$, the off-axis light curve approaches the on-axis one, since for $\Gamma \theta_{\text {obs }} \ll 1, a \approx b \approx 1$ and, as a result, $F_{\nu}\left(\theta_{\mathrm{obs}}, t_{\mathrm{obs}}\right) \approx F_{\nu}\left(0, t_{\mathrm{obs}}\right)$.

This simple model of a point source along the jet symmetry axis (called model 1 in Figure 11.9) is instructive, as it captures much of the qualitative behavior of off-axis light curves. Nevertheless, it is important to also consider more realistic jet models. A useful model is a uniform jet of half-opening 

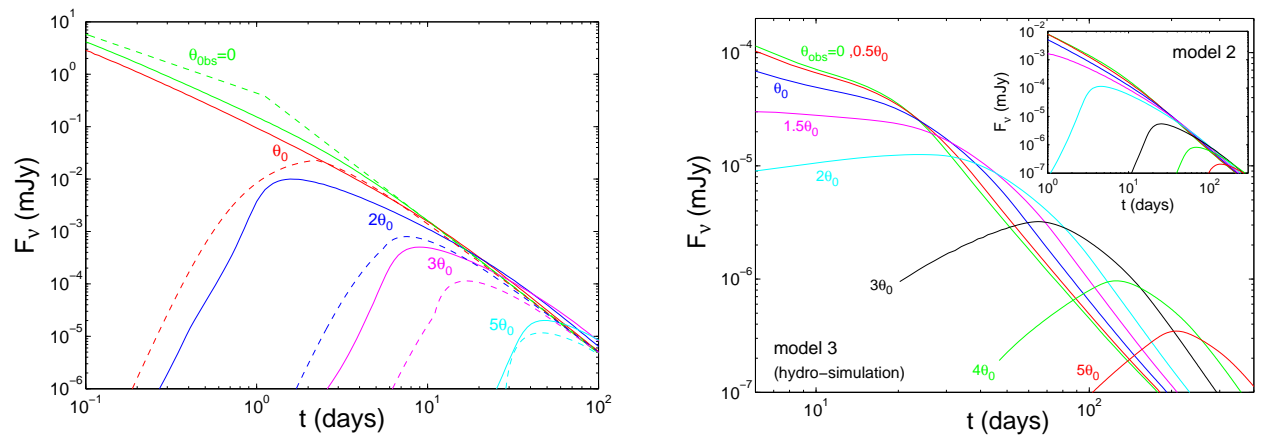

Fig. 11.9. Afterglow light curves for different viewing angles (from Granot et al. 2002): Left panel: comparing a simple analytic model featuring a point source along the jet axis (model 1; dashed lines) and a uniform jet with sharp edges that expands laterally at the sound speed in the jet comoving frame (model 2; solid lines). Right panel: comparing model 2 (inset) to the light curves calculated from a 2D hydrodynamical simulation of an initially uniform jet with sharp edges.

angle $\theta_{\mathrm{j}}$, with an initial value $\theta_{0}$, which expands laterally at some speed $\beta_{\mathrm{s}} c$ in its own, comoving rest frame (where $\beta_{\mathrm{s}}=0$ corresponds to no lateral expansion and $\beta_{\mathrm{s}} \sim 1$ corresponds to relativistic expansion in the comoving frame). For model 2 in Figure 11.9, $\beta_{\mathrm{s}} c$ is taken to be the local sound speed, which implies $\beta_{\mathrm{s}} \approx 1 / \sqrt{3}$ during the relativistic stage. The dynamics are given by conservation of energy and rest mass. The light curves for model 2 differ considerably from those for model 1 for $\theta_{\text {obs }} \lesssim \theta_{0}$ at early times. This is because in model 2 , for such viewing angles, the observed flux at early times is dominated by material along the line of sight, whose beaming cone encompasses the line of sight from the very beginning, while in model 1 there is no emission along the line of sight, and the emission from the point source along the jet axis is strongly beamed away from the observer. Moreover, in model 2 the light curves for $\theta_{\text {obs }} \leq \theta_{0}$ are very similar to those for $\theta_{\mathrm{obs}}=0$, since the observed emission is mainly from an angle of $1 / \Gamma$ around the line of sight, and for such viewing angles this region is mostly (or even totally) occupied by the uniform jet, resulting in very small differences in the observed flux density.

We note that model 1 can be made somewhat more realistic by placing the point source at the edge of the jet at the point $\mathrm{P}$ closest to the line of sight for viewing angles outside of the jet aperture, i.e., using $\theta=\max \left(0, \theta_{\mathrm{obs}}-\theta_{\mathrm{j}}\right)$ instead of $\theta=\theta_{\text {obs }}$ for the angle between the point source and the line of sight. In model 2 , for viewing angles $1 / \Gamma_{0} \ll \theta_{\text {obs }}-\theta_{0} \ll \theta_{0}$ at early times when $\Gamma\left(\theta_{\text {obs }}-\theta_{\mathrm{j}}\right) \gg 1$ the emission is dominated by the part of the jet that is closest to the line of sight. Such emission arises from the area within the 
jet where the Doppler factor is close to its highest value (at point $\mathrm{P}$ ), which is over a solid angle of the order of $\sim\left(\theta_{\mathrm{obs}}-\theta_{\mathrm{j}}\right)^{2}$. Therefore, if there were no lateral spreading, the initial rise in the flux would be similar to that for a point source at a fixed angle from the line of sight (which was discussed earlier). If, however, the jet spreads laterally, $\theta_{\mathrm{obs}}-\theta_{\mathrm{j}}$ would decrease in time, but the effect of the reduction in the fraction of the jet contributing to the observed emission is overwhelmed by the more rapid increase in flux due to the faster rate at which the beaming cone of the emitting material approaches the line of sight.

For the prompt emission, the ratio of $E_{\text {peak }}$ - the photon energy at which the $\nu F_{\nu}$ spectrum peaks - between lines of sight outside the jet $\left(\theta_{\text {obs }}>\theta_{0}\right)$ and those within the jet (which are similar to $\theta_{\text {obs }}=0$ ) is $a \approx 1 /[1+$ $\left.\Gamma^{2}\left(\theta_{\text {obs }}-\theta_{0}\right)^{2}\right] \sim\left[\Gamma\left(\theta_{\text {obs }}-\theta_{0}\right)\right]^{-2}$. The ratio of their apparent isotropic equivalent energy, $E_{\gamma, \text { iso }}$, which is the ratio of their observed fluence, is $\sim a^{2}$ for viewing angles that are relatively close to the edge of the jet, $1 / \Gamma_{0}<$ $\theta_{\text {obs }}-\theta_{0}<\theta_{0}$ (Eichler \& Levinson 2004), since in this case only a fraction $\sim\left(\theta_{\text {obs }}-\theta_{0}\right)^{2} / \theta_{0}^{2} \propto 1 / a$ of the jet (in the part closest to the line of sight) contributes significantly to the observed emission, resulting is a suppression by a factor of $\sim 1 / a$ relative to the result of $a^{3}$ for a point source. For a roughly standard $E_{\text {peak }}$ at viewing angles within the jet, this reproduces the observed correlation $E_{\text {peak }} \propto E_{\gamma, \text { iso }}^{1 / 2}$, reported by Amati et al. (2002), Lloyd-Ronning \& Ramirez-Ruiz (2002), and subsequently Lamb et al. (2005) using data from BeppoSAX, BATSE and HETE-2, respectively. $\dagger$ For larger viewing angles, $\theta_{\text {obs }} \gtrsim 2 \theta_{0}$, we have $E_{\gamma \text {,iso }}\left(\theta_{\text {obs }}\right) / E_{\gamma, \text { iso }}(0) \sim\left(\Gamma \theta_{0}\right)^{2}\left[\Gamma\left(\theta_{\text {obs }}-\right.\right.$ $\left.\left.\theta_{0}\right)\right]^{-6} \sim\left(\Gamma \theta_{0}\right)^{-4}\left(\theta_{\mathrm{obs}} / \theta_{0}\right)^{-6}$, which scales as $a^{3} \sim\left(\Gamma \theta_{\mathrm{obs}}\right)^{-6}$. This is similar to the expected change of $E_{\gamma \text {,iso }}$ with viewing angle for a point source, since for such viewing angles all of the jet contributes similarly to the observed emission, and it may be reasonably approximated as a point source.

A more accurate description of the jet dynamics can be achieved with hydrodynamic simulations. The resulting light curves for an initially uniform jet with sharp edges, for different viewing angles, are shown in the right panel of Figure 11.9 (model 3). The initially sharp edges of the jet quickly become smoother and the jet becomes non-uniform, especially near the edges, were there is a sharp decrease in the Lorentz factor and energy per solid angle, and the velocity is not in the radial direction. This results in a much larger contribution to the observed flux at early times for large viewing angles

$\uparrow$ This empirical correlation has inspired many theoretical investigations arguing for its origin despite the many debates on its observational validity. The reader is referred to Gehrels et al. (2009) for a review. 


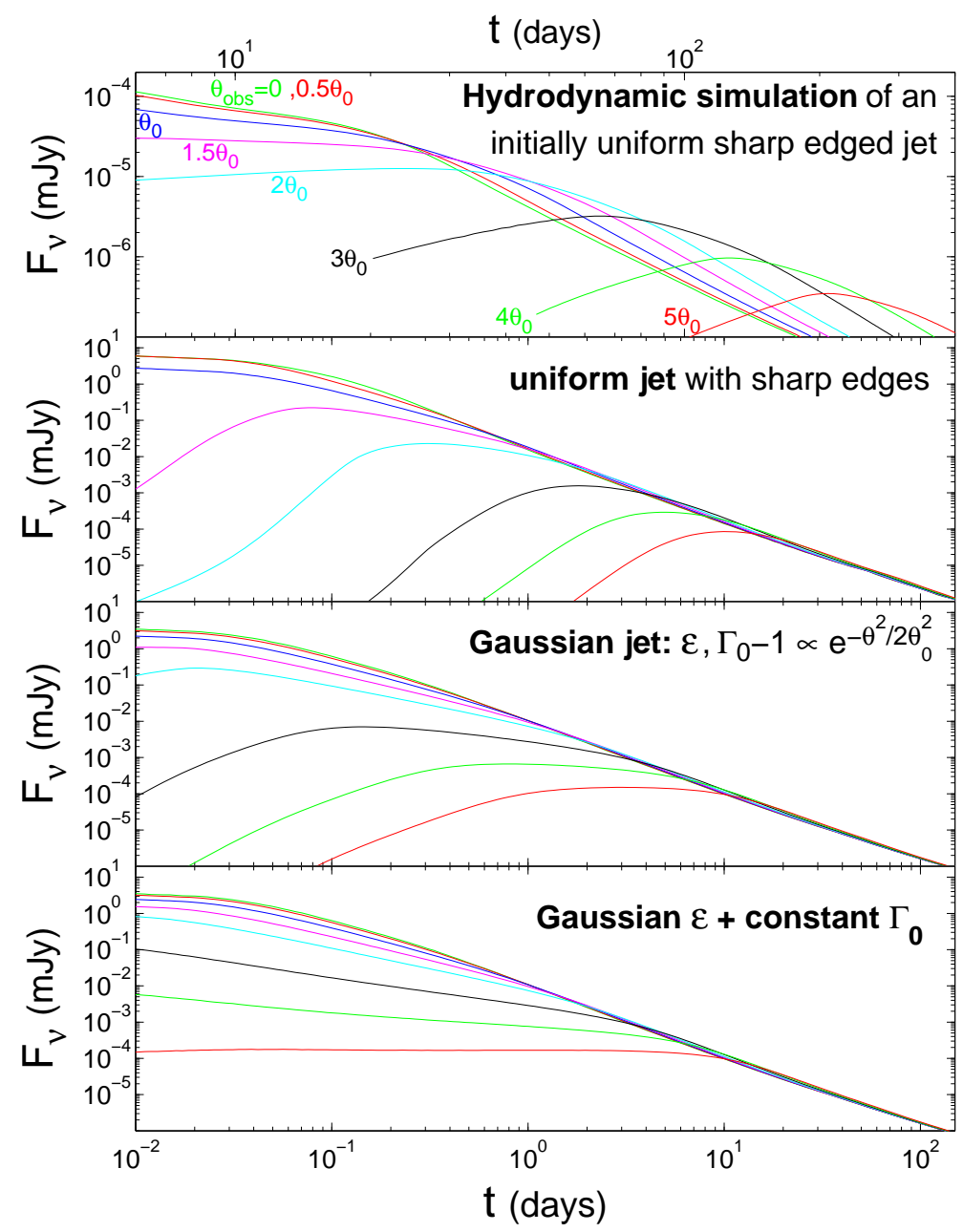

Fig. 11.10. Afterglow light curves for different jet structures, dynamics, and viewing angles (from Eichler \& Granot 2006). The top panel is from an initially uniform jet with sharp edges whose evolution is calculated using a hydrodynamic simulation, the second panel is for a uniform jet with sharp edges, and the two bottom panels are for a Gaussian jet, in energy per solid angle, and either a Gaussian or a uniform initial Lorentz factor. The viewing angles are $\theta_{\text {obs }} / \theta_{0}=0,0.5,1,1.5,2,3,4,5$, where $\theta_{0}$ is the (initial) half-opening angle for the uniform jet (two top panels) and the core angle $\left(\theta_{c}\right)$ for the Gaussian jet (two bottom panels).

outside the jet initial aperture, which in turn gives a slower rise in flux compared to a perfectly uniform jet with sharp edges (such as model 2).

The light curves for different viewing angles depend not only on the jet dynamics but also on its initial angular profile, and in particular on $\Gamma_{0}(\theta)$ 
and $\mathcal{E}(\theta)$ (where $\mathcal{E}$ is the jet energy per solid angle). Figure 11.10 compares the light curves for different viewing angles, between different jet structures. The first panel from the top is for model 3 described above, the second panel is for a uniform jet with no lateral expansion, while the last two panels are for a Gaussian $\mathcal{E}(\theta)$, with either a Gaussian or a constant $\Gamma_{0}(\theta)$. For a Gaussian jet, if both $\mathcal{E}(\theta)$ and $\Gamma_{0}(\theta)$ have a Gaussian profile (corresponding to a constant rest mass per solid angle in the outflow), then the afterglow light curves are rather similar to those for a uniform jet (Kumar \& Granot 2003). If, on the other hand, $\mathcal{E}(\theta)$ is Gaussian while $\Gamma_{0}(\theta)=$ const, then the light curves for off-axis viewing angles (i.e., outside the core of the jet) have a much higher flux at early times, compared to a Gaussian $\Gamma_{0}(\theta)$ or a uniform jet (see the bottom two panels of Figure 11.10), due to a dominant contribution from the emitting material along the line of sight which has an early deceleration time in this case (Granot et al. 2005).

\subsection{Unification schemes: implications of viewing angle effects}

The appearance of GRBs depends so strongly on their orientation relative to us that our current classification schemes might easily be dominated by our random viewing angles rather than by more interesting (intrinsic) physical properties. These inherently highly anisotropic GRB models imply a radically different GRB appearance at different viewing angles. In practice, GRBs of different orientations will thus likely be assigned to different classes. Unification of these fundamentally identical but apparently disparate classes is an essential stepping stone to studying the underlying physical properties of GRBs. The ultimate goal is to discover which are the fundamentally important characteristics of GRBs - e.g., black hole mass, black hole spin, accretion rate - and how they govern the formation of jets, and the production of radiation. In this section we thus critically examine GRB off-axis models and contrast them with the afterglow observations of X-ray flashes and sub-luminous long GRBs. Since afterglow data in these cases are too sparse and insufficient to derive meaningful constraints on the overall population, we concentrate our efforts on a few well-monitored examples.

Empirical classification of GRBs: GRBs traditionally have been assigned to different classes based on their duration. On the basis of this criterion, there are two classes of GRBs - short and long - dividing at $\sim 2 \mathrm{~s}$ duration (Kouveliotou et al. 1993). GRBs have also been classified according to their spectral properties, albeit less successfully. In particular, bursts with lower photon energies $E_{\text {peak }}$ at which the $\nu F_{\nu}$ spectrum peaks have 
been dubbed X-ray flashes (XRFs) based on observations by BeppoSAX, BATSE, and HETE-2 (Heise et al. 2001, Barraud et al. 2003, Kippen et al. 2003, Lamb et al. 2004, Sakamoto et al. 2005). XRFs, with durations ranging from several seconds to a few minutes and a distribution on the sky consistent with being isotropic, are similar to long duration ( $\gtrsim 2 \mathrm{~s})$ GRBs. In addition to XRFs, the empirical classification of variable X-ray transients observed by HETE-2 had been expanded to include an intermediate class of events known as X-ray rich GRBs (XRGRBs). The spectrum of XRGRBs and XRFs is similar to that of long GRBs (Sakamoto et al. 2005) except for their lower values of $E_{\text {peak }}$ and of $E_{\gamma \text {,iso }}$ (their X-ray and/or gamma-ray energy output assuming isotropic emission). In all respects XRFs, XRGRBs and long GRBs seem to form a smooth continuum.

Many different models have been proposed for XRFs, most of which try to incorporate them into a unified scenario with GRBs. These models include high-redshift GRBs (Heise et al. 2001), dirty (low- $\left.\Gamma_{0}\right)$ fireballs (Dermer et al. 1999, Huang et al. 2002, Zhang et al. 2003), regular GRBs viewed off-axis (Yamazaki et al. 2002, Dado et al. 2004, Kouveliotou et al. 2004), photosphere-dominated emission (Drenkhahn 2002, Ramirez-Ruiz \& LloydRonning 2002, Mészáros et al. 2002), weak internal shocks (low variability, $\Delta \Gamma \ll \Gamma$; Zhang \& Mészáros 2002, Barraud et al. 2003, Mochkovitch et al. 2004), and large viewing angles in a structured (Lamb et al. 2005) or quasiuniversal (Zhang et al. 2004) jet. Most of these models mainly aim at explaining the low values of $E_{\text {peak }}$ in XRFs and do not address their expected afterglow properties. The afterglow evolution alone can, however, serve as a powerful test for XRF models. In fact, most of the models discussed for XRFs and XRGRBs have at least one major flaw in common: they do not naturally produce the very flat afterglow light curve seen at early times. In what follows we thus concentrate only on the class of models that naturally produce such light curves, that is, a roughly uniform jet with sufficiently sharp edges viewed from outside of the jet core. This class of models has been discussed quantitatively in Sect. 11.10, Since most afterglow data of XRFs and XRGRBs are too sparse, here we critically examine the role of off-axis models and contrast them with afterglow observations of XRGRB 041006 and GRB 031203 for which the afterglow light curves are reasonably well monitored from sufficiently early times. The reader is referred to Swift observations of XRF 080330 (Guidorzi et al. 2009) and GRB 081028 (Margutti et al. 2009) for more recent examples of XRFs or GRBs, which bear similar characteristics to the ones discussed here.

Off axis jet models of XRGRBs and XRFs: The case of 041006 . 
XRGRB 041006 was detected by HETE-2 (Galassi et al. 2004). It had a fluence of $5 \times 10^{-6} \mathrm{erg} \mathrm{cm}^{-2}$ in the $2-30 \mathrm{keV}$ range and $7 \times 10^{-6} \mathrm{erg} \mathrm{cm}^{-2}$ in the $30-400 \mathrm{keV}$ range, corresponding to $f_{X / \gamma} \approx-0.15$ which classifies it as an XRGRB. It has a redshift of $z=0.716$ (Fugazza et al. 2004), which for a fluence of $f \approx 1.2 \times 10^{-5} \mathrm{erg} \mathrm{cm}^{-2}$ in the $2-400 \mathrm{keV}$ range gives $E_{\gamma \text {,iso }} \approx 1.6 \times 10^{52} \mathrm{erg}$. It had an observed peak photon energy of $E_{\text {peak }}^{\text {obs }}=63_{-5}^{+7} \mathrm{keV}$, corresponding to $E_{\text {peak }}^{\text {rest }}=109_{-9}^{+12} \mathrm{keV}$. Figure11.11 shows an off-axis model yielding an acceptable fit to the to the optical and X-ray afterglow observations of XRGRB 041006, which is also consistent with the upper limits at radio and sub-mm wavelengths (Granot et al. 2005). From this analysis one can conclude that a successful model for the afterglow of XRGRB 041006 is that of a collimated, misaligned jet interacting with a stellar wind external medium of mass density $\rho_{\text {ext }}=A r^{-2}$, where $r$ is the distance from the central source. The parameter values used in this fit are: $E=1.0 \times 10^{51} \mathrm{erg}, A_{*} \equiv A /\left(5 \times 10^{11} \mathrm{gr} \mathrm{cm}^{-1}\right)=0.03, \theta_{0}=3^{\circ}, \theta_{\mathrm{obs}}=1.15 \theta_{0}$, $p=2.2, \epsilon_{e}=0.1$, and $\epsilon_{B}=0.001$.

The optical light curve is very flat at early times $(\alpha \sim 0$ at $t \lesssim 1 \mathrm{hr}$, where $\left.F_{\nu} \propto t^{-\alpha} \nu^{-\beta}\right)$ and becomes steeper after a few hours $(\alpha \approx 1.2)$, which is a little steeper than the decay index in $\mathrm{X}$ rays at a similar time $(\alpha \approx 1$ at $t \approx 1$ day). Also, the ratio of the flux in optical and $\mathrm{X}$ rays at $t \approx 1$ day implies a spectral index of $\beta \approx 0.7-0.75$ assuming a single power law between them. This suggests that the cooling break frequency $\nu_{c}$ is above the optical after 1 day. Since one requires very extreme parameters to get $\nu_{c}$ to the X-ray range after 1 day (even getting $\nu_{c}$ to be above the optical after a day requires relatively low values of $\epsilon_{B}$ and of the external density), it is most likely that $\nu_{c}$ is between the optical and X-ray at 1 day, which can also explain the steeper temporal decay index in the optical (by $\Delta \alpha=0.25$ ) for a stellar wind environment $(k=2)$. This favors a wind medium over a uniform density one, since otherwise the flux in the optical will decay more slowly than in the X-ray (also by $\Delta \alpha=0.25$ ), which is contrary to what is observed for XRGRB 041006. At $t \gtrsim 5$ days there is a flattening in the optical light curve, which is probably due to an underlying SN component or host. This explains why the observed flux is higher than that predicted by our narrow relativistic jet model.

For a GRB jet with well-defined edges, both the prompt gamma-ray fluence and the peak of the spectrum drop very sharply outside the opening of the jet, as $a^{2}$ (or $a^{3}$ depending on $\theta_{\text {obs }}$ ) and $a$, respectively. Therefore, the low $E_{\gamma, \text { iso }}$ of XRGRB 041006 combined with $E_{\mathrm{k}, \text { iso }}=E /\left(1-\cos \theta_{0}\right) \approx$ $E\left(2 / \theta_{0}^{2}\right) \approx 7.3 \times 10^{53} \mathrm{erg}$ (which serves as a proxy for the $E_{\gamma, \text { iso }}$ value that would have been measured by an on-axis observer) implies $\left(E_{\mathrm{k}, \text { iso }} / E_{\gamma, \text { iso }}\right)^{1 / 2} \sim$ 


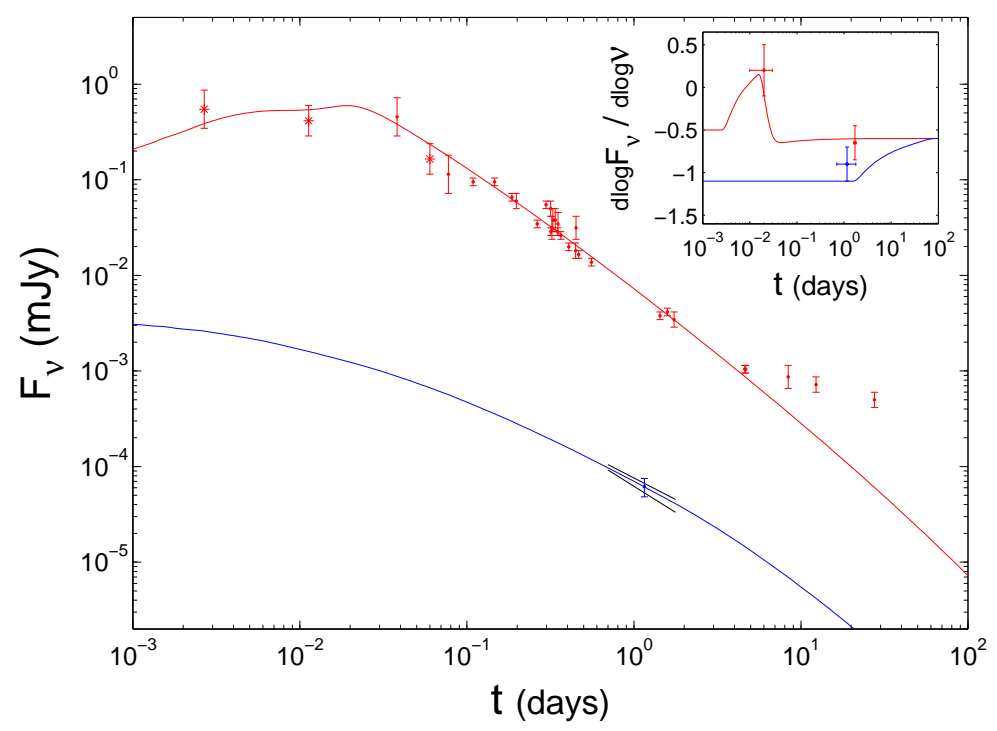

Fig. 11.11. A tentative fit to the optical R-band (upper curve) and X-ray (0.5 $6 \mathrm{keV}$, lower curve) data of XRGRB 041006 (see Granot et al. 2005 and references therein). The ROTSE-IIIa points are shown with asterisk symbols since they are unfiltered, but they can still be treated as R-band observations within the measurement errors. We also added two lines to the X-ray data which indicate the edges of the $1 \sigma$ confidence interval for the temporal decay index, $\alpha=1 \pm 0.1$, and cover the duration of the Chandra observation. The inset shows the predicted spectral slope, $-\beta=d \log F_{\nu} / d \log \nu$, in the optical (upper curve) and in the X-ray (lower curve), together with the values inferred from observations.

6.8 and $\Gamma \sim\left(E_{\mathrm{k}, \text { iso }} / E_{\gamma, \text { iso }}\right)^{1 / 4}\left(\theta_{\text {obs }}-\theta_{0}\right)^{-1} \sim 330$. This implies a (cosmological) rest frame on-axis $E_{\text {peak }}\left(\theta_{\text {obs }}<\theta_{0}\right) \sim\left(E_{\mathrm{k} \text {,iso }} / E_{\gamma, \text { iso }}\right)^{1 / 2} E_{\text {peak }} \sim 740 \mathrm{keV}$, which falls closely within the observed $E_{\text {peak }}-E_{\gamma \text {,iso }}$ relation.

Off-axis jet models for sub-luminous GRBs: the case of 031203 . At a relatively small distance, with a redshift of $z=0.1055$ (Prochaska et al. 2004), GRB 031203 was also atypical in its isotropic equivalent gammaray energy output, with $E_{\gamma \text {,iso }} \sim 10^{50} \mathrm{ergs}$ (Sazonov et al. 2004). In fact its $E_{\gamma \text {,iso }}$ was intermediate between GRB 980425 and more typical bright GRBs with $E_{\gamma \text {,iso }} \sim 10^{52}-10^{54}$ ergs (Frail et al. 2001). The gamma-ray light curve was smooth and similar to GRB 980425, consisting of a single peak lasting about $20 \mathrm{~s}$ and a peak photon energy $E_{\text {peak }}>190 \mathrm{keV}$. Soon afterwards, an optical counterpart was identified and follow-up observations by several telescopes revealed a supernova, SN 2003lw, with a spectrum very similar to that of SN 1998bw (e.g., Malesani et al. 2004). Subsequent 
X-ray observations of GRB 031203 with $X M M$ and Chandra identified an $\mathrm{X}$-ray source coincident with the optical transient. The flux decay rate and the isotropic luminosity of the X-ray afterglow also ranked the event as intermediate between GRB 980425 and classical GRBs (Kouveliotou et al. 2004). A very faint counterpart was also detected at centimeter wavelengths where it displayed a peak luminosity more than two orders of magnitude fainter than typical radio afterglows (Frail et al. 2003), but again comparable to that of GRB 980425.

GRB 031203, or at least its gamma-ray luminosity directed at us, was certainly very weak. A straightforward interpretation might be that the GRB was deficient in all its emissions in all directions. This idea is compatible with the afterglow light curve at radio frequencies. However, when one combines the fact that a 20 s long GRB was observed, as well as an X-ray and infrared afterglow, the situation is more constrained and in fact more consistent with a model in which GRB 031203 was a typical powerful jetted GRB viewed off-axis (Ramirez-Ruiz et al. 2005).

The off-axis jet interpretation for GRB 031203 requires the viewing angle to have been $\theta_{\text {obs }} \sim 2 \theta_{0}$ (Figure 11.12). A misaligned jet with a typical energy expanding into a stellar wind with properties similar to those of WolfRayet stars is thus consistent with the observations, especially with the slow initial decline rates seen in both the $\mathrm{X}$-ray and radio afterglow. One question that naturally arises is whether the observed gamma-ray flux of GRB 031203 can be explained within the framework of this model. The low $E_{\gamma, \text { iso }}$ of GRB 031203 implies $\dagger \theta_{0}=3.8^{\circ}\left(E_{\gamma, \text { iso }} / 10^{50} \mathrm{erg}\right)^{-1 / 8}\left(E_{\text {jet }} / 3 \times 10^{50} \mathrm{erg}\right)^{1 / 8}$ $(\Gamma \Upsilon / 50)^{-3 / 4}$, where $E_{\text {jet }}$ is the kinetic energy of the jet, and $\Upsilon=\theta_{\text {obs }} / \theta_{0}-1$ and the fiducial values were chosen to match those of GRB 031203, which were either observed $\left(E_{\gamma \text {,iso }} \sim 10^{50} \mathrm{erg}\right)$ or inferred from the fit to its afterglow $\left(\theta_{0} \sim 3^{\circ}-5^{\circ}, E_{\text {jet }} \sim 3 \times 10^{50} \mathrm{erg}, \Upsilon \approx 1\right)$. A consistent solution for both afterglow and prompt emission can thus be found if $\Gamma \sim 50$ and $\Upsilon \approx 1$, which imply more typical values of $E_{\mathrm{p}} \sim 2 \mathrm{MeV}$ (given the observed value $E_{\text {peak }} \sim 190 \mathrm{keV}$ ) and $E_{\gamma \text {,iso }} \sim 10^{53} \mathrm{ergs}$ when observed on-axis (consistent within the intrinsic spread of the $E_{\text {peak }}-E_{\gamma \text {,iso }}$ relation). These results are applicable in the present context provided only that one further condition is satisfied, namely, that the (on-axis) jetted outflow be optically thin to high-energy photons. For a burst with $E_{\text {peak }} \sim 2 \mathrm{MeV}, \Gamma$ must exceed $\sim 50$. We thus conclude that the observations, especially the slow initial decline rates seen in the X-ray afterglow, are more consistent with an off-axis model in which GRB 031203 was a much more powerful GRB seen at an angle of 


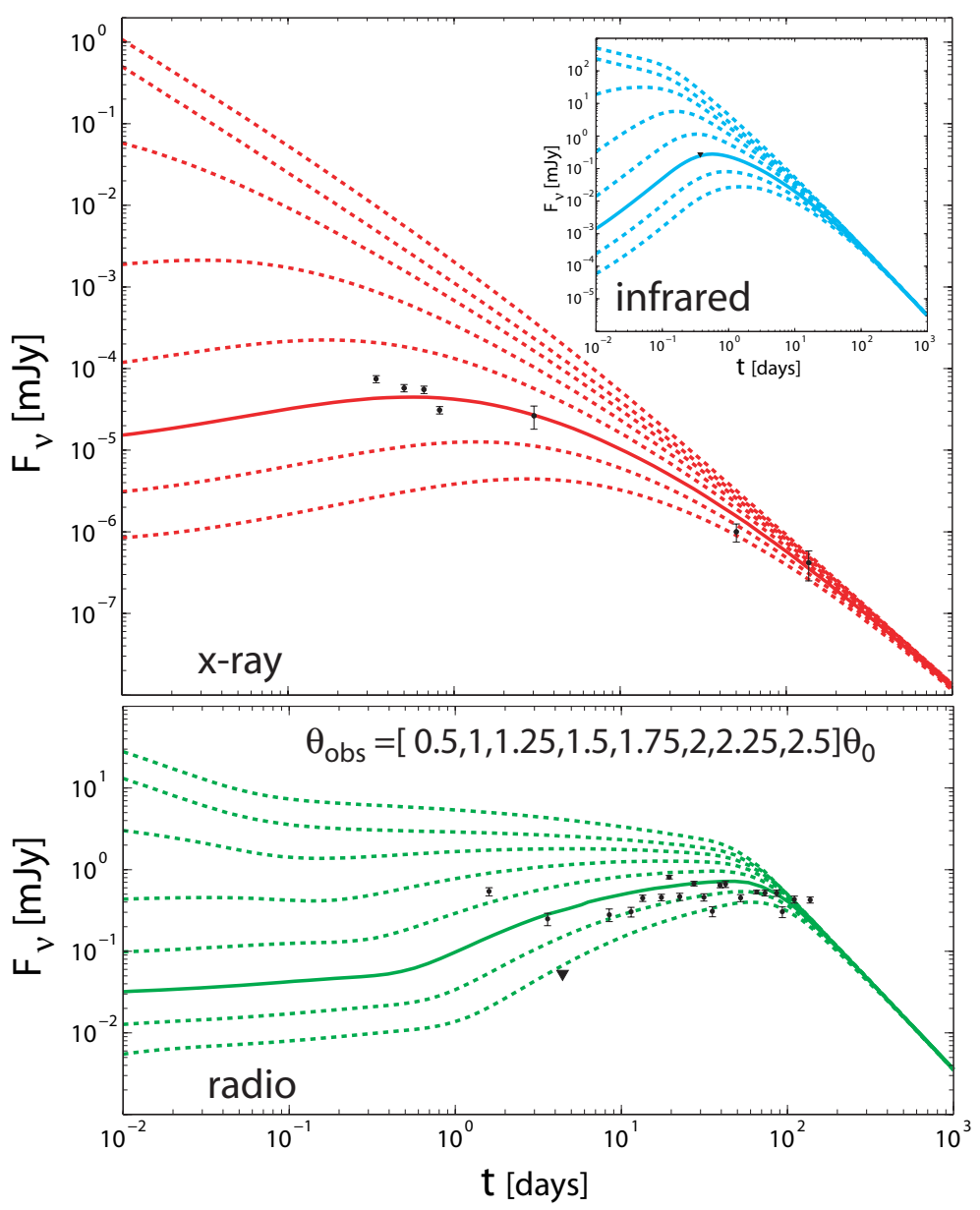

Fig. 11.12. Afterglow emission from a sharp edged uniform jet in GRB 031203. Light curves are calculated for various viewing angles $\theta_{\text {obs }}$ for a GRB with the standard parameters $E_{\text {jet }}=3 \times 10^{50} \mathrm{erg}, p=2.4, \epsilon_{e}=0.15, \epsilon_{B}=0.02, \theta_{0}=5^{\circ}$, and $A_{*}=\left(\dot{M} / 10^{-5} \mathrm{M}_{\odot} \mathrm{yr}^{-1}\right)\left(v_{w} / 10^{3} \mathrm{~km} \mathrm{~s}^{-1}\right)^{-1}=0.1$. The data for GRB 031203 can be reasonably fit by different sets of model parameters (i.e., the parameters cannot be uniquely determined by the data). For example, a sharp-edged jet with $\theta_{0}=3.5^{\circ}$ seen at $\theta_{\text {obs }} \approx 2.25 \theta_{0}$ gives also a reasonably good description of the observations provided that $\epsilon_{e}=0.1$ and $\epsilon_{B}=0.04$.

about two times the opening angle of the central jet.

Unified schemes for GRBs: The empirical classification scheme by which an event is tagged as a GRB, sub-luminous GRB, XRGRB or XRF is rather arbitrary. Therefore, there could be some cases where a jet that is viewed 
on-axis $\left(\theta_{\text {obs }}<\theta_{0}\right)$ will be classified as an XRGRB or XRF instead of as a GRB, or the opposite case in which a jet viewed off-axis $\left(\theta_{\text {obs }}>\theta_{0}\right)$ might be classified as a GRB instead of as an XRGRB or an XRF. A more physically motivated classification would be according to the ratio of the viewing angle $\theta_{\text {obs }}$ and the jet half-opening angle $\theta_{0}$, instead of relying purely on spectral characteristics as in the present empirical scheme. Such a classification would, however, be much harder to implement as it is not a trivial task to accurately determine the viewing angle. The strongest constraints could thus be obtained from afterglow light curves of XRFs an XRGRBs that are well monitored from early times and at various frequencies (ranging from radio to X-rays). Current examples include Swift observations of XRF 080330 (Guidorzi et al. 2009) and GRB 081028 (Margutti et al. 2009)

Similarly, the large statistical sample of GRBs and XRFs with redshift measurements will allow a reconstruction of the intrinsic luminosity function of the prompt emission. If GRBs, XRGRBs and XRFs are only a manifestation of the viewing angle for a structured, universal jet (whose wings are producing the XRFs), then no break would be expected in the luminosity function. On the other hand, if GRBs are the results of viewing angles that intersect the jet (whether structured or not), while XRFs and XRGRBs are off-axis events, then one would naturally expect a break in the luminosity function. Guetta et al. (2005) found that a luminosity function with a break is favored in order for the predicted rate of local bursts to be consistent with the observed rate. This also prevents the existence of an exceedingly large number of GRB remnants in the local Universe.

The relative fraction of XRFs and XRGRBs to GRBs is also expected to be different in the various models. If indeed an XRF corresponds to $\Gamma\left(\theta_{\text {obs }}-\theta_{0}\right) \sim$ a few and $\left(\theta_{\text {obs }}-\theta_{0}\right) \lesssim \theta_{0}$, then the solid angle from which an XRF is seen scales as $\theta_{0} / \Gamma$ or as $\theta_{0}$ for a constant $\Gamma$ (at a constant distance to the source), while the solid angle from which a GRB is seen scales as $\theta_{0}^{2}$. Therefore, the ratio of solid angles for GRBs and XRFs scales as $\theta_{0}$, and more GRBs compared to XRFs would be seen for larger $\theta_{0}$. As the distance to the source increases, XRFs could be detected only out to a smaller offaxis viewing angle, while most GRBs would still be bright enough to be detected out to reasonably large redshifts. Therefore, the ratio of GRBs to $\mathrm{XRFs}$ should increase with redshift. Finally, if the true energy $E$ in the jet is roughly constant, then the maximal redshift out to which a GRB could be detected would decrease with $\theta_{0}$ since $E_{\gamma \text {,iso }} \propto \theta_{0}^{-2}$. This would increase the statistical weight of narrow jets in an observed sample, as they could be seen out to a larger volume.

Finally, we conclude with a few possible implications of the off-axis model 
hypothesis for XRFs and XRGRBs. For sufficiently large viewing angles outside the edge of the jet, one might expect some decrease in the variability of the prompt emission. This is because the width of an individual spike in the light curve scales as $\Delta t \propto 1 / a$ while the peak photon energy and fluence scale as $a$ and $a^{2}-a^{3}$, respectively. Since the interval between neighboring spikes in the light curve is typically comparable to the width of an individual spike, $\Delta t$, then if $\Delta t$ increases significantly for large viewing angles this would cause at least some overlap between different pulses which would smear out some of the variability. Thus one might expect XRFs to be somewhat less variable than GRBs, at least on average, where a lower variability might be expected for lower values of $E_{\text {peak }}$ and $E_{\gamma \text {,iso. This }}$ may lead to a simple physical interpretation of the observed variabilityluminosity relation in the prompt gamma-ray/X-ray emission (Fenimore \& Ramirez-Ruiz 2000, Reichart et al. 2001).

Another possible signature of the off-axis model for XRFs and XRGRBs is in the reverse shock emission. If the reverse shock is at least mildly relativistic, then the optical flash emission would be less beamed than the prompt $\mathrm{X}$-ray or gamma-ray emission, due to the deceleration of the ejecta by the passage of the reverse shock. This might cause the optical flash to be suppressed by a smaller factor relative to the gamma-ray emission, compared to the corresponding on-axis fluxes. Thus XRFs or XRGRBs might still show reasonably bright optical emission from the reverse shock, which might in some cases be almost as bright as for classical GRBs. Finally, XRFs and XRGRBs might also show a larger degree of polarization compared to GRBs.

Our understanding of GRBs has come a long way since their discovery over 40 years ago, but these enigmatic sources continue to offer major puzzles and challenges. As we have described, our rationalization of the principal physical considerations for the prompt and afterglow radiation emanating from these objects combines some generally accepted features with some more speculative ingredients. When confronted with observations, it seems to accommodate their gross features but fails to provide us with a fully predictive theory - but then again, no such theory exists as of yet. What is more valuable, though considerably harder to achieve, is to refine models like the ones advocated here to the point of making clearer quantitative predictions, and to assemble, assess and interpret observations so as to constrain or refute these theories. What we can hope of our present understanding is that it will assist us in such an endeavor. 
Acknowledgments J.G and E.R. gratefully acknowledge support from a Royal Society Wolfson Research Merit Award and a David and Lucile Packard Fellowship, respectively.

\section{References}

Abdo, A.A. et al. (2009a). Science 323, 1688.

Abdo, A.A. et al. (2009b). ApJ 706, L138.

Ackermann, M. et al. (2010). ApJ 716, 1178.

Amati, L. et al. (2002). $A \mathscr{E} A$ 390, 81.

Baring, M.G. \& Harding, A.K. (1997). ApJ 491, 663.

Barraud, C. et al. (2003). A\&A 400, 1021.

Berger, E. et al. (2003). Nat 426, 154.

Berger, E., Kulkarni, S.R., \& Frail, D.A. (2004). ApJ 612, 966.

Blandford, R.D. \& McKee, C.F. (1976). Phys. Fluids 19, 1130.

Blustin, A.J. et al. (2006). ApJ 637, 901.

Burrows, D. N., et al. (2009). in Conf. Proc., Chandra's First Decade of Discovery, ed. by S. Wolk, A. Fruscione, and D. Swartz, abstract \#23

Cannizzo, J.K., Gehrels, N., \& Vishniac, E.T. (2004). ApJ 601, 380.

Coburn, W. \& Boggs, S.E. (2003). Nat 423, 415.

Cohen, E. \& Piran, T. (1999). ApJ 518, 346.

Covino, S., Ghisellini, G., Lazzati, D., \& Malesani, D. (2004). in Gamma-Ray Bursts in the Afterglow Era, ed. M. Feroci, F. Frontera, N. Masetti, \& L. Piro. (San Francisco: ASPC), 312, 169.

Dado, S., Dar, A., \& De Rujula, A. (2004). A\& A 422, 381.

Dai, X., et al. (2008). ApJ 682, L77.

Dermer, C.D., Chiang J., \& Böttcher, M. (1999). ApJ 513, 656.

Drenkhahn, G. (2002). A\&A 387, 714.

Eichler, D. \& Granot, J. (2006). ApJ 641, L5.

Eichler, D. \& Levinson, A. (2003). ApJ 596, L147.

Eichler, D. \& Levinson, A. (2004). ApJ 614, L13.

Eichler, D. \& Waxman, E. (2005). ApJ 627, 861.

Fenimore, E.E., Epstain, R.I., \& Ho, C. (1993). A\&AS 97, 59.

Fenimore, E.E., \& Ramirez-Ruiz, E. (2000). astro-ph/0004176.

Firmani, C., Avila-Reese, V., Ghisellini, G., \& Tutukov, A.V. (2004). ApJ 611, 1033.

Fong, W., Berger, E., \& Fox, D.B. (2010). ApJ 708, 9.

Frail, D.A. et al. (1997). Nat 389, 261.

Frail, D.A. et al. (2000). ApJ 538, L129.

Frail, D.A. et al. (2001). ApJ 562, L55.

Frail, D.A. et al. 2003) $A J$ 125, 2299.

Frail, D.A. et al. (2005). ApJ 619, 994.

Fruchter, A.S. et al. (1999). ApJ 519, L13.

Fugazza, D. et al. (2004). GCN Circ. 2782.

Galama, T.J. et al. (1998). ApJ 500, L97.

Galassi, M. et al. (2004) GCN Circ. 2770.

Garnavich, P.M., Loeb, A., \& Stanek, K.Z. (2000). ApJ 544, L11.

Gaudi, B.S., Granot, J., \& Loeb, A. (2001). ApJ 561, 178.

Gehrels, N. et al. (2005). Nat 437, 851. 
Gehrels, N. et al. (2008). ApJ 689, 1161.

Gehrels, N., Ramirez-Ruiz, E., \& Fox, D.B. (2009). ARA\&A 47, 567.

Ghisellini, G., \& Lazzati, D. (1999). MNRAS 309, L7.

Goodman, J. (1997). New Astron. 2, 449.

Götz, D. et al. (2009). ApJ 695, L208.

Granot, J. (2003). ApJ 596, L17.

Granot, J. (2005). ApJ 631, 1022.

Granot, J. (2007). Rev. Mex. A\&A 27, 140.

Granot, J. (2008). MNRAS 390, L46.

Granot, J., Cohen-Tanugi, J., \& do Couto e Silva, E. (2008). ApJ 677, 92.

Granot, J., Königl, A., \& Piran, T. (2006). MNRAS 370, 1946.

Granot, J. \& Königl, A. (2003). ApJ 594, L83.

Granot, J. \& Kumar, P. (2003). ApJ 591, 1086.

Granot, J. et al. (2001). in "GRBs in the Afterglow Era", ed. E. Costa, F. Frontera, \& J. Hjorth (Berlin: Springer), 312.

Granot, J. \& Loeb, A. (2001). ApJ 551, L63.

Granot, J., Panaitescu, A., Kumar, P., \& Woosley, S.E. (2002). ApJ 570, L61.

Granot, J., Piran, T., \& Sari, R. (1999a). ApJ 527, 236.

Granot, J., Piran, T., \& Sari, R. (1999b). ApJ 513, 679.

Granot, J., Ramirez-Ruiz, E. \& Loeb, A. (2005). ApJ 618, 413.

Granot, J., Ramirez-Ruiz, E., \& Perna, R. (2005). ApJ 630, 1003.

Granot, J. \& Sari, R. (2002). ApJ 568, 820.

Greiner, J. et al. (2003). Nat 426, 157.

Gruzinov, A. \& Waxman, E. (1999). ApJ 511, 852.

Guetta, D., Granot, J., \& Begelman, M.C. (2005). ApJ 622, 482.

Guetta, D., Piran, T., \& Waxman, E. (2005). ApJ 619, 412.

Guidorzi, C. et al. (2009). $A \mathscr{E} A$ 499, 439.

Halpern, J.P. et al. (1999). ApJ 517, L105.

Halpern, J.P. et al. (2000). ApJ 543, 697.

Harrison, F.A. et al. (1999). ApJ 523, L121.

Harrison, F.A. et al. (2001). ApJ 559, 123.

Heise, J., in 't Zand, J., Kippen, R.M., \& Woods, P.M. (2001). in Gamma-Ray Bursts in the Afterglow Era, ed. E. Costa, F. Frontera, \& J. Hjorth (Berlin: Springer), 16.

Hjorth, J. et al. (2003). Nat 423, 847.

Huang, Y.F., Dai, Z.G., \& Lu, T. (2002). MNRAS 332, 735.

Huang, Y.F., Wu, X.F., Dai, Z.G., Ma, H.T., \& Lu, T. (2004). ApJ 605, 300.

Katz, J. I., \& Piran, T. (1997). ApJ 490, 772.

Kippen, R.M. et al. (2003). in AIP Conf. Proc. 662, Gamma-Ray Bursts and Afterglow Astronomy, ed. G.R. Ricker \& R.K. Vanderspek (New York: AIP), 244.

Kocevski, D. \& Butler, N. (2008). ApJ 680, 531.

Kouveliotou, C. et al. (1993). ApJ 413, L101.

Kouveliotou, C. et al. (2004). ApJ 608, 872.

Krolik, J.H. \& Pier, E.A. (1991). ApJ 373, 277.

Kulkarni, S.R. et al. (1999). Nat 398, 389.

Kumar, P. \& Granot, J. (2003). ApJ 591, 1075.

Kumar, P. \& Panaitescu, A. (2000). ApJ 541, L9.

Kumar, P. \& Piran, T. (2000). ApJ 535, 152.

Lamb, D.Q. et al. (2004). New Astron. Rev. 48, 423. 
Lamb, D.Q., Donaghy, T.Q., \& Graziani, C. (2005). ApJ 620, 355.

Lazzati, D., \& Begelman, M. C. (2005). ApJ 629, 903.

Lazzati, D., Rossi, E., Ghisellini, G., \& Rees, M.J. (2004). MNRAS 347, L1.

Levinson, A. \& Eichler, D. (1993). ApJ 418, 386.

Levinson, A. \& Eichler, D. (2000, PRL 85, 236.

Liang, E.-W., et al. (2008). ApJ 675, 528.

Lipunov, V.M., Postnov, K.A., \& Prokhorov, M.E. (2001). Astron. Rep. 45, 236.

Lithwick, Y. \& Sari, R. (2001). ApJ 555, 540.

Lloyd-Ronning, N.M. \& Ramirez-Ruiz, E. (2002). ApJ 576, 101.

Loeb, A. \& Perna, R. (1998). ApJ 495, 597.

Lyutikov, M., Pariev, V.I., \& Blandford, R.D. (2003). ApJ 597, 998.

Malesani, J. et al. (2004). ApJ 609, L5.

Margutti, R. et al. (2009). MNRAS 402, 46.

McGlynn, S. et al. (2007). A\&A 466, 895.

Medvedev, M.V. \& Loeb, A. (1999). ApJ 526, 697.

Mészáros, P., Ramirez-Ruiz, E., Rees, M.J., \& Zhang, B. (2002). ApJ 578, 812.

Mészáros, P., Rees, M.J., \& Wijers, R.A.M.J. (1998). ApJ 499, 301.

Mochkovitch, R., Daigne, F., Barraud, C., \& Atteia, J.L. (2004). in Gamma-Ray Bursts in the Afterglow Era, ed. M. Feroci, F. Frontera, N. Masetti, \& L. Piro (San Francisco: ASP), 381.

Moderski, R., Sikora, M., \& Bulik, T. (2000). ApJ 529, 151.

Nakar, E., Granot, J., \& Guetta, D. (2004). ApJ 606, L37.

Nakar, E. \& Oren, Y. (2004). ApJ 602, L97.

Nakar, E., Piran, T. \& Waxman, E. (2003). JCAP 10, 005.

Nousek, J.A. et al. (2006). ApJ 642, 389.

Panaitescu, A. \& Mészáros, P. (1999). ApJ 526, 707.

Paragi, Z. et al. (2010). Nat 463, 516.

Pedersen, H. et al. (1998). ApJ 496, 311.

Peng, F., Königl, A., \& Granot, J. (2005). ApJ 626, 966.

Perna, R., Sari, R., \& Frail, D. (2003). ApJ 594, 379.

Perna, R. \& Vietri, M. (2002). ApJ 569, L47.

Pihlström, Y.M., Taylor, G.B., Granot, J., \& Doeleman, S. (2007). ApJ 664, 411.

Piran, T. (2000). Phys. Rep. 333, 529.

Price, P.A. et al. (2001). ApJ 549, L7.

Prochaska, J.X. et al. (2004). ApJ 611, 200.

Racusin, J.L. et al. (2008). Nat 455, 183.

Racusin, J.L. et al. (2009). ApJ 698, 43.

Ramirez-Ruiz, E., Celotti, A., \& Rees, M.J. (2002). MNRAS 337, 1349.

Ramirez-Ruiz, E., Granot, J., Kouveliotou, C., Woosley, S.E., Patel, S.K., \& Mazzali, P.A. (2005). ApJ 625, L91.

Ramirez-Ruiz, E. \& Lloyd-Ronning, N.M. (2002). New Astron. 7, 197.

Ramirez-Ruiz, E. \& Madau, E. (2004). ApJ 608, L89.

Ramirez-Ruiz, E., Merloni, A., \& Rees, M.J. (2001). MNRAS 324, 1147.

Reichart, D., Lamb, D., Fenimore, E.E., Ramirez-Ruiz, E., Cline, T., \& Hurley, K. (2001). ApJ 552, 57.

Rhoads, J.E. (1997). ApJ 487, L1.

Rhoads, J.E. (1999). ApJ 525, 737.

Rol, E. et al. (2003). A\&A 405, L23.

Rossi, E., Lazzati, D., \& Rees, M.J. (2002). MNRAS 332, 945.

Rossi, E., Lazzati, D., Salmonson, J.D., \& Ghisellini, G. (2004). MNRAS 354, 86. 
Ruderman, M. (1975). Ann. NY Acad. Sci. 262, 164.

Rybicki, G.B., \& Lightman, A.P. (1976, "Radiative Processses in Astrophysics", Wiley Interscience, p.145.

Rutledge, R.E. \& Fox, D.E. (2004). MNRAS 350, 1288.

Sagar, R. et al. (2001). Bull. Astron. Soc. India 29, 1.

Sakamoto, T. et al. (2005). ApJ 629, 311.

Sari, R. (1997). ApJ 489, L37.

Sari, R. (1998). ApJ 494, L49.

Sari, R. (1999). ApJ 524, L43.

Sari, R. \& Esin, A.A. (2001). ApJ 548, 787.

Sari, R. \& Mésáros, P. (2000). ApJ 535, L33 .

Sari, R. \& Piran, T. (1995). ApJ 455, L143.

Sari, R., Piran, T., \& Halpern, J. (1999). ApJ 519, L17.

Sazonov, S.Y., Lutovinov, A.A., \& Sunyaev, R.A. (2004). Nat 430, 646.

Shaviv, N.J. \& Dar, A. (1995). ApJ 447, 863.

Soderberg, A.M., Nakar, E., Berger, E., \& Kulkarni, S.R. (2006). ApJ 638, 930.

Soderberg, A.M. et al. (2010). Nat 463, 513.

Stanek, K.Z. et al. (2003). ApJ 591, L17.

Stanek, K.Z. et al. (2007). ApJ 654, L21 .

Tan, J.C., Matzner, C.D., \& McKee, C.F. (2001). ApJ 551, 946.

Tanvir, N. R., et al. (2010). ApJ 725, 625.

Taylor, G.B., Frail, D.A., Beasley, A.J., \& Kulkarni, S.R. (1997). Nat 389, 263.

Taylor, G.B., Frail, D.A., Berger, E., \& Kulkarni, S.R. (2004). ApJ 609, L1.

Taylor, G.B., et al. (2005). ApJ 622, 986.

Vlahakis, N., Peng, F., \& Königl, A. (2003). ApJ 594, L23.

Waxman, E. (2003). Nat 423, 388.

Waxman, E., Kulkarni, S.R., \& Frail, D.A. (1998). ApJ 497, 288.

Wigger, C. et al. (2004). ApJ 613, 1088.

Wijers, R.A.M.J. \& Galama, T.J. (1999). ApJ 523, 177.

Willingale, R. et al. (2007). ApJ 662, 1093.

Willis, D.R. et al. (2005). A\&A 439, 245.

Woods, E. \& Loeb, A. (1995). ApJ 453, 583.

Yamazaki, R., Ioka, K., \& Nakamura, T. (2002). ApJ 571, L31.

Yamazaki, R., Ioka, K., \& Nakamura, T. (2004). ApJ 607, L103.

Zeh, A., Klose, S., \& Kann, D.A. (2006). ApJ 637, 889.

Zhang, B. \& Mészáros, P. (2002). ApJ 571, 876.

Zhang, B., Dai, X., Lloyd-Ronning, N.M., \& Mészáros, P. (2004). ApJ 601, L119.

Zhang, W., Woosley, S.E., \& MacFadyen, A.I. (2003). ApJ 586, 356.

Zhang, W. \& MacFadyen, A. (2009). ApJ 698, 1261. 